\title{
On the Complexity of Nash Equilibria in Anonymous Games
}

\author{
Xi Chen \\ Columbia University
}

\author{
David Durfee \\ Georgia Institute of Technology
}

\author{
Anthi Orfanou \\ Columbia University
}

\begin{abstract}
We show that the problem of finding an $\epsilon$-approximate Nash equilibrium in an anonymous game with seven pure strategies is complete in PPAD, when the approximation parameter $\epsilon$ is exponentially small in the number of players.
\end{abstract}

\section{Introduction}

The celebrated theorem of Nash [Nas50, Nas51] states that every game has an equilibrium point. The concept of Nash equilibrium has been tremendously influential in economics and social sciences ever since (e.g., see [HR04]), and its computation has been one the most well-studied problems in the area of Algorithmic Game Theory. For normal form games with a bounded number of players, much progress has been made during the past decade in understanding both the complexity of Nash equilibrium [AKV05, CDT06, CTV07,DGP09, CDT09, Meh14] as well as its efficient approximation [LMM04, BVV05, KPS06, DMP06, DMP07, BBM07, KT07, TS07, FNS07, KS07, ABOV07, TS10].

In this paper we study a large and important class of succinct multiplayer games called anonymous games (see [Sch73, Mil96, Blo99, Blo05, Kal05] for studies of such games in the economics literature). These are special multiplayer games in that the payoff of each player depends only on (1) the pure strategy of the player herself, and (2) the number of other players playing each pure strategy, instead of the full pure strategy profile. In such a game, the (expected) payoff of a player is highly symmetric over (pure or mixed) strategies of other players. For instance, two players switching their strategies would not affect the payoff of any other player. A consequence of this very special payoff structure is that $O\left(\alpha n^{\alpha-1}\right)$ numbers suffice to completely describe the payoff function of a player, when there are $\alpha$ pure strategies shared by $n$ players. Notably this is polynomial in the number of players when $\alpha$ is bounded, and hence the game is succinctly representable. Throughout the paper, we focus on succinct anonymous games with a bounded number of pure strategies.

Other well-studied multiplayer games with a succinct representation include graphical, symmetric, and congestion games (for more details see [PR08]). While graphical and congestion games are both known to be hard to solve [FPT04,ARV08,SV08], there is indeed a polynomial-time algorithm for computing an exact Nash equilibrium in a symmetric game [PR08]. Because anonymous games generalize symmetric games by allowing player-dependent payoff functions, it is a natural question to ask whether there is an efficient algorithm for finding an (exact or approximate) Nash equilibrium in an anonymous game as well.

Culminating in a sequence of beautiful papers [DP07,Das08,DP08,DP09, DP14] Daskalakis and Papadimitriou obtained a polynomial-time approximation scheme (PTAS) for $\epsilon$-approximate Nash equilibria in anonymous games with a bounded number of strategies (see more discussion on related 
work in Section 1.1). However, the complexity of finding an exact Nash equilibrium in such games remains open, and was conjectured to be hard for PPAD in [DP09,DP14]. ${ }^{1}$

In this paper we give an affirmative answer to the conjecture of Daskalakis and Papadimitriou, by showing that it is PPAD-complete to find an $\epsilon$-approximate Nash equilibrium in an anonymous game, when the approximation parameter $\epsilon$ is exponentially small in $n$. To formally state our main result, let $(\alpha, c)$-ANONYMOUs denote the problem of finding a $\left(2^{-n^{c}}\right)$-approximate Nash equilibrium in an anonymous game with $\alpha$ pure strategies and payoffs from $[0,1]{ }^{2}$

Here is our main theorem:

Theorem 1. For any $\alpha \geq 7$ and $c>0$, the problem $(\alpha, c)$-AnOnymous is PPAD-complete.

The greatest challenge to establishing the PPAD-completeness result stated above is posed by the rather complex but also highly symmetric payoff structure of anonymous games. Before discussing our approach and techniques in Section 1.3, we first review related work in Section 1.1, then define anonymous games formally and introduce some useful notation in Section 1.2.

\subsection{Related Work}

Anonymous games have been studied extensively in the economics literature [Sch73, Ras83, Mil96, Blo99, Blo05, Kal05, ES05], where the game being considered is usually nonatomic and consists of a continuum of players but a finite number of strategies. For the discrete setting, two special families of anonymous games are symmetric games [PR08, BFH09] and congestion games [Ros73]. [PR08] gave a polynomial-time for finding an exact Nash equilibrium in a symmetric game. For congestion games, PLS-completeness of pure equilibria was established in [FPT04,ARV08,SV08 ${ }^{3}$, and efficient approximation algorithms for various latency functions were obtained in [CFGS11, CFGS12, CS11].

While an anonymous game does not possess a pure Nash equilibrium in general, it was shown in [DP07, AS11,DP14] that when the payoff functions are $\lambda$-Lipschitz, there exists an $\epsilon$-approximate pure Nash equilibrium and it can be found in polynomial time, where $\epsilon$ has a linear dependency on $\lambda$. Furthermore, in [Bab13] Babichenko presented a best-reply dynamic for $\lambda$-Lipschitz anonymous games with two strategies which reaches an approximate pure equilibrium in $O(n \log n)$ steps.

Regarding our specific point of interest, i.e., (mixed) Nash equilibria in anonymous games with a scaling number of players but a non-scaling number of strategies, there have been a sequence of positive and negative results obtained by Daskalakis and Papadimitriou [DP07,DP08, Das08,DP09] (summarized in the journal version [DP14]). We briefly review these results below.

In [DP07], Daskalakis and Papadimitriou presented a PTAS for finding an $\epsilon$-approximate Nash equilibrium in an anonymous game with two pure strategies, with running time $n^{O\left(1 / \epsilon^{2}\right)} \cdot U$, where $U$ denotes the number of bits required to describe the payoffs. The running time was subsequently improved in [Das08] to poly $(n) \cdot(1 / \epsilon)^{O\left(1 / \epsilon^{2}\right)} \cdot U$. The first PTAS in [DP07] is based on the existence of an $\epsilon$-approximate Nash equilibrium consisting of integer multiples of $\epsilon^{2}$, while the second PTAS in [Das08] is based on the existence of an $\epsilon$-approximate Nash equilibrium satisfying the following

\footnotetext{
${ }^{1}$ When the number of pure strategies is a sufficiently large constant, an anonymous game with rational payoffs may not have any rational equilibrium (e.g., by embedding in it a rational three-player game with no rational equilibrium). But for the case of two strategies, it remains unclear as whether every rational anonymous game has a rational Nash equilibrium, which was posed as an open problem in [DP14].

${ }^{2}$ Since we are interested in the additive approximation, all payoffs are normalized to take values in $[0,1]$.

${ }^{3}$ These PLS-hardness results have no implication to the setup of this paper since the number of pure strategies in the congestion games considered there are unbounded.
} 
special property: either at most $O\left(1 / \epsilon^{3}\right)$ players play mixed strategies, or all players who mix play the same mixed strategy. Later [DP08] extended the result of [DP07], giving the only known PTAS for anonymous games with any bounded number of pure strategies with time $n^{g(\alpha, 1 / \epsilon)} \cdot U$ for some function $g$ of $\alpha$, number of pure strategies, and $1 / \epsilon$.

All three PTAS obtained in [DP07,Das08,DP08] are so-called oblivious algorithms [DP09], i.e., algorithms that enumerate a set of mixed strategy profiles that is independent of the input game as candidates for approximate Nash equilibria (hence, the game is used only to verify if a given mixed strategy profile is an $\epsilon$-approximate Nash equilibrium). In [DP09], Daskalakis and Papadimitriou showed that any oblivious algorithm for anonymous games must have running time exponential in $1 / \epsilon$. In contrast to this negative result, they also presented a non-oblivious PTAS for two-strategy anonymous games with running time $\operatorname{poly}(n) \cdot(1 / \epsilon)^{O\left(\log ^{2}(1 / \epsilon)\right)} \cdot U$.

\subsection{Anonymous Games and Polymatrix Games}

Before giving a high-level description of our approach and techniques in Section 1.3, we first give a formal definition of anonymous games and introduce some useful notation. Consider a multiplayer game with $n$ players $[n]=\{1, \ldots, n\}$ and $\alpha$ pure strategies $[\alpha]=\{1, \ldots, \alpha\}$ with $\alpha$ being a constant. For each pure strategy $b \in[\alpha]$, let $\psi_{b}(\mathbf{t})$ denote the number of $b$ 's in a tuple $\mathbf{t} \in[\alpha]^{n-1}$, and define $\Psi(\mathbf{t})=\left(\psi_{1}(\mathbf{t}), \ldots, \psi_{\alpha}(\mathbf{t})\right)$, which we will refer to as the histogram of pure strategies in $\mathbf{t}$.

In an anonymous game, the payoff of each player $p \in[n]$ depends only on $\Psi\left(\mathbf{s}_{-p}\right)$ and her own strategy $s_{p}$, given a pure strategy profile $\mathbf{s} \in[\alpha]^{n}$. (We follow the convention and use $\mathbf{s}_{-p} \in[\alpha]^{n-1}$ to denote the pure strategy profile of the $n-1$ players other than player $p$ in s.) Informally, $\Psi\left(\mathbf{s}_{-p}\right)$ can be described as what player $p$ "sees" in the game when $\mathbf{s}$ is played.

We now formally define anonymous games.

Definition 2. An anonymous game $\mathcal{G}=\left(n, \alpha,\left\{\right.\right.$ payoff $\left.\left._{p}\right\}\right)$ consists of a set $[n]$ of $n$ players, a set $[\alpha]$ of $\alpha$ pure strategies, and a payoff function payoff $p:[\alpha] \times K \rightarrow \mathbb{R}$ for each player $p \in[n]$, where

$$
K=\left\{\left(k_{1}, \ldots, k_{\alpha}\right): k_{j} \in \mathbb{Z}_{\geq 0} \text { for all } j \text { and } \sum_{j=1}^{\alpha} k_{j}=n-1\right\}
$$

is the set of all histograms of pure strategies played by $n-1$ players. Specifically, when $\mathbf{s} \in[\alpha]^{n}$ is played, the payoff of player $p$ is given by payoff $_{p}\left(s_{p}, \Psi\left(\mathbf{s}_{-p}\right)\right)$.

As usual, a mixed strategy is a probability distribution $\mathbf{x}=\left(x_{1}, \ldots, x_{\alpha}\right)$, and a mixed strategy profile $\mathcal{X}$ is an ordered tuple of $n$ mixed strategies $\left(\mathbf{x}_{p}: p \in[n]\right)$, one for each player $p$. Given $\mathcal{X}$, let $u_{p}(b, \mathcal{X})$ denote the expected payoff of $p$ playing $b \in[\alpha]$, which has the following explicit expression:

$$
u_{p}(b, \mathcal{X})=\sum_{\mathbf{k} \in K} \text { payoff }_{p}(b, \mathbf{k}) \cdot \operatorname{Pr}_{\mathcal{X}}[p, \mathbf{k}]
$$

where $\operatorname{Pr}_{\mathcal{X}}[p, \mathbf{k}]$ denotes the probability of player $p$ seeing histogram $\mathbf{k}$ under $\mathcal{X}$ :

$$
\operatorname{Pr}_{\mathcal{X}}[p, \mathbf{k}]=\sum_{\mathbf{s}_{-p} \in \Psi^{-1}(\mathbf{k})}\left(\prod_{q \neq p} x_{q, s_{q}}\right) .
$$

Note that $s_{q}$ denotes the pure strategy of player $q$ from a profile $\mathbf{s}_{-p} \in \Psi^{-1}(\mathbf{k})$. We also use $u_{p}(\mathcal{X})$ to denote the expected payoff of player $p$ from playing $\mathbf{x}_{p}$ :

$$
u_{p}(\mathcal{X})=\sum_{b \in[\alpha]} x_{p, b} \cdot u_{p}(b, \mathcal{X})
$$


It is worth pointing out that, while $u_{p}(b, \mathcal{X})$ contains exponentially many terms, it can be computed in polynomial time using dynamic programming [DP07,DP14] when $\alpha$ is a constant. For a detailed presentation of the algorithm for 2-strategy anonymous games, see [DP14]. This then implies that checking whether a given profile $\mathcal{X}$ is a (approximate) Nash equilibrium is in polynomial time.

Next we define (approximate) Nash equilibria of an anonymous game.

Definition 3. Given an anonymous game $\mathcal{G}=\left(n, \alpha,\left\{\right.\right.$ payoff $\left.\left._{p}\right\}\right)$, we say a mixed strategy profile $\mathcal{X}$ is a Nash equilibrium of $\mathcal{G}$ if $u_{p}(\mathcal{X}) \geq u_{p}(b, \mathcal{X})$ for all players $p \in[n]$ and strategies $b \in[\alpha]$.

For $\epsilon \geq 0$, we say $\mathcal{X}$ is an $\epsilon$-approximate Nash equilibrium if $u_{p}(\mathcal{X})+\epsilon \geq u_{p}(b, \mathcal{X})$ for all $p \in[n]$ and $b \in[\alpha]$. For $\epsilon \geq 0$, we say $\mathcal{X}$ is an $\epsilon$-well-supported Nash equilibrium if $u_{p}(a, \mathcal{X})+\epsilon<u_{p}(b, \mathcal{X})$ implies that $x_{p, a}=0$, for all $p \in[n]$ and $a, b \in[\alpha]$.

As discussed in Section 1.3, the hardness part of Theorem 1 is proved using a polynomial-time reduction from the problem of finding a well-supported Nash equilibrium in a polymatrix game (e.g. see [CD11]). For our purposes, such a game (with $n$ players and two strategies each player) can be described by a payoff matrix $\mathbf{A} \in[0,1]^{2 n \times 2 n}$ with $A_{k, \ell}=0$ for all $k, \ell \in\{2 i-1,2 i\}$ and $i \in[n]$.

Each player $i \in[n]$ has two pure strategies that correspond to rows $2 i-1$ and $2 i$ of $\mathbf{A}$. Let $\mathbf{A}_{j}$ denote the $j$ th row of $\mathbf{A}$. Given a vector $\mathbf{y} \in \mathbb{R}_{\geq 0}^{2 n}$, where $\left(y_{2 i-1}, y_{2 i}\right)$ is the mixed strategy of player $i$, expected payoffs of player $i$ for playing rows $2 i-1$ and $2 i$ are $\mathbf{A}_{2 i-1} \cdot \mathbf{y}$ and $\mathbf{A}_{2 i} \cdot \mathbf{y}$ respectively.

An $\epsilon$-well-supported Nash equilibrium of $\mathbf{A}$ is a vector $\mathbf{y} \in \mathbb{R}_{\geq 0}^{2 n}$ such that $y_{2 i-1}+y_{2 i}=1$ and

$$
\mathbf{A}_{2 i-1} \cdot \mathbf{y}>\mathbf{A}_{2 i} \cdot \mathbf{y}+\epsilon \Rightarrow y_{2 i}=0 \quad \text { and } \quad \mathbf{A}_{2 i} \cdot \mathbf{y}>\mathbf{A}_{2 i-1} \cdot \mathbf{y}+\epsilon \Rightarrow y_{2 i-1}=0,
$$

for all players $i \in[n]$. We need the following result on such games:

Theorem 4 ([CPY13]). The problem of computing a $(1 / n)$-well-supported Nash equilibrium in a polymatrix game is PPAD-complete.

\subsection{Our Approach and Techniques}

A commonly used approach to establishing the PPAD-hardness of approximate equilibria is to design gadget games that can perform certain arithmetic operations on entries of mixed strategies of players (e.g. see [DGP09, CDT09]). Such gadgets would then yield a reduction from the problem of solving a generalized circuit [DGP09, CDT09], a problem complete in PPAD. However, we realized that this approach may not work well with anonymous games; we found that it was impossible to design an anonymous game $G_{=}$that enforces equality constraints. ${ }^{4}$

Instead we show the PPAD-hardness of anonymous games via a reduction from the problem of finding a $(1 / n)$-well-supported equilibrium in a two-strategy polymatrix game (see Section 1.2). Given a $2 n \times 2 n$ polymatrix game $\mathbf{A}$, our reduction constructs an anonymous game $\mathcal{G}_{\mathbf{A}}$ with $n$ "main" players $\left\{P_{1}, \ldots, P_{n}\right\}$ (and two auxiliary players). We have each main player $P_{i}$ simulate in a way a player $i$ in the polymatrix game, as discussed below, such that any $\epsilon$-well-supported Nash equilibrium of $\mathcal{G}_{\mathbf{A}}$ with an exponentially small $\epsilon$ can be used to recover a $(1 / n)$-well-supported Nash equilibrium of the polymatrix game A efficiently. We then prove a connection between approximate Nash equilibria and well-supported Nash equilibria of anonymous games to finish the proof of Theorem 1.

\footnotetext{
${ }^{4}$ For example, we can rule out the existence of an anonymous game $G=$ with 4 players and 2 pure strategies such that $\mathbf{x}$ is a Nash equilibrium of $G_{=}$if and only if $x_{1}=x_{2} \in[\mu, \nu] \subseteq[0,1]$ and $x_{3}=x_{4} \in\left[\mu^{\prime}, \nu^{\prime}\right] \subseteq[0,1]$, where we use $x_{i}$ to denote the probability that player $i$ plays the first pure strategy.
} 
The greatest challenge to establishing such a reduction is posed by the complex but highly structured, symmetric expression of expected payoffs in an anonymous game. As discussed previously in Section 1.2, the expected payoff $u_{p}(b, \mathcal{X})$ of player $p$ is a linear form of probabilities $\operatorname{Pr}_{\mathcal{X}}[p, \mathbf{k}]$, each of which is function over mixed strategies of all players other than $p$. This rather complex function makes it difficult to reason about the set of well-supported Nash equilibria of an anonymous game, not to mention our goal is to embed a polymatrix game in it. To overcome this obstacle, we need to find a special (but hard enough) family of anonymous games with certain payoff structures which allow us to perform a careful analysis and understand their well-supported equilibria. The bigger obstacle for our reduction, however, is to in some sense remove the anonymity of the players and break the inherent symmetry underlying an anonymous game.

To see this, a natural approach to obtain a reduction from polymatrix games is to directly encode the $2 n$ variables of $\mathbf{y}$ in mixed strategies of the $n$ "main" players $\left\{P_{1}, \ldots, P_{n}\right\}$. More specifically, let $\left\{s_{1}, s_{2}\right\}$ denote two special pure strategies of $\mathcal{G}_{\mathbf{A}}$, and we attempt to encode $\left(y_{2 i-1}, y_{2 i}\right)$ in $\left(x_{i, s_{1}}, x_{i, s_{2}}\right)$, probabilities of $P_{i}$ playing $s_{1}, s_{2}$, respectively. The reduction would work if expected payoffs of $P_{i}$ from $s_{1}$ and $s_{2}$ in $\mathcal{G}_{\mathbf{A}}$ can always match closely expected payoffs of player $i$ from rows $2 i-1$ and $2 i$ in $\mathbf{A}$, given by two linear forms $\mathbf{A}_{2 i-1} \cdot \mathbf{y}$ and $\mathbf{A}_{2 i} \cdot \mathbf{y}$ of $\mathbf{y}$. However, it seems difficult, if not impossible, to construct $\mathcal{G}_{\mathbf{A}}$ with this property, since anonymous games are highly symmetric: the expected payoff of $P_{i}$ is a symmetric function over mixed strategies of all other players. This is not the case for polymatrix games: a linear form such as $\mathbf{A}_{2 i} \cdot \mathbf{y}$ in general has different coefficients for different variables, so different players contribute with different weights to the expected payoff of a player (and the problem of finding a well-supported equilibrium in $\mathbf{A}$ clearly becomes trivial if we require that every row of $\mathbf{A}$ has the same entry).

An alternative approach is to encode the $2 n$ variables of $\mathbf{y}$ in probabilities $\operatorname{Pr}_{\mathcal{X}}[p, \mathbf{k}]$. This may look appealing because expected payoffs $u_{p}(b, \mathcal{X})$ are linear forms of these probabilities so one can set the coefficients payoff $(b, \mathbf{k})$ to match them easily with those linear forms $\mathbf{A}_{j} \cdot \mathbf{y}$ that appear in the polymatrix game $\mathbf{A}$. However, the histogram $\mathbf{k}$ seen by a player $p$ (as a vector-valued random variable) is the sum of $n-1$ vector-valued random variables, each distributed according to the mixed strategy of a player other than $p$. The way these probabilities $\operatorname{Pr}_{\mathcal{X}}[p, \mathbf{k}]$ are derived in turn imposes strong restrictions on them, ${ }^{5}$ which makes it a difficult task to obtain a correspondence between the $2 n$ free variables in $\mathbf{y}$ and the probabilities $\operatorname{Pr} \mathcal{X}[p, \mathbf{k}]$.

Our reduction indeed follows the first approach of encoding $\left(y_{2 i-1}, y_{2 i}\right)$ in $\left(x_{i, s_{1}}, x_{i, s_{2}}\right)$ of player $P_{i}$. More exactly, the former is the normalization of the latter into a probability distribution. Now to overcome the difficulty posed by symmetry, we enforce the following "scaling" property in every well-supported Nash equilibrium $\mathcal{X}$ of $\mathcal{G}_{\mathbf{A}}$ : probabilities of $P_{i}$ playing $\left\{s_{1}, s_{2}\right\}$ satisfy

$$
x_{i, s_{1}}+x_{i, s_{2}} \approx 1 / N^{i}
$$

where $N$ is exponentially large in $n$. This property is established by designing an anonymous game called generalized radix game $\mathcal{G}_{n, N}^{*}$, and then using it as the base game in the construction of $\mathcal{G}_{\mathbf{A}}$. We show that (1) holds approximately for every anonymous game that is payoff-wise close to $\mathcal{G}_{n, N}^{*}$. In particular, (1) holds for any well-supported equilibrium of $\mathcal{G}_{\mathbf{A}}$, as long as we make sure $\mathcal{G}_{\mathrm{A}}$ is close to $\mathcal{G}_{n, N}^{*}$. The "scaling" property plays a crucial role in our reduction because, as the base game for $\mathcal{G}_{\mathbf{A}}$, it helps us reason about well-supported Nash equilibria of $\mathcal{G}_{\mathbf{A}}$; it also removes

\footnotetext{
${ }^{5}$ For example, as it is pointed out in [DP07, DP08] for anonymous games with two strategies, players can always be partitioned into a few sets such that the probabilities $\operatorname{Pr}_{\mathcal{X}}[p, \mathbf{k}]$ over $\mathbf{k}$ must follow approximately a Poisson or a discretized Normal distribution on each set respectively.
} 
anonymity of the $n$ "main" players $P_{i}$ (since they must play the two special pure strategies $\left\{s_{1}, s_{2}\right\}$ with probabilities of different scales) and overcome the symmetry barrier.

Equipped with the "scaling" property (1), we prove a key technical lemma called the estimation lemma. It shows that one can compute efficiently coefficients of a linear form over probabilities of histograms $\operatorname{Pr}_{\mathcal{X}}\left[P_{i}, \mathbf{k}\right]$ seen by player $P_{i}$, which guarantees to approximate additively $x_{j, s_{1}}$ (or $x_{j, s_{2}}$ ) i.e. probability of another player $P_{j}$ plays $s_{1}$ (or $s_{2}$ ), whenever the profile $\mathcal{X}$ satisfies the "scaling" property (this holds when $\mathcal{G}_{\mathbf{A}}$ is close to $\mathcal{G}_{n, N}^{*}$ and $\mathcal{X}$ is a well-supported equilibrium of $\mathcal{G}_{\mathbf{A}}$ ). As

$$
\left(y_{2 j-1}, y_{2 j}\right) \approx N^{j}\left(x_{j, s_{1}}, x_{j, s_{2}}\right)
$$

given (1), these linear forms for $x_{j, s_{1}}, x_{j, s_{2}}$ can be combined to derive a linear form of $\operatorname{Pr}_{\mathcal{X}}\left[P_{i}, \mathbf{k}\right]$ to approximate additively any linear form of $\mathbf{y}$, particularly $\mathbf{A}_{2 i-1} \cdot \mathbf{y}$ or $\mathbf{A}_{2 i} \cdot \mathbf{y}$ that appear as expected payoffs of player $i$ in the polymatrix game $\mathbf{A}$. The proof of the estimation lemma is the technically most involved part of the paper. We indeed derive explicit expressions for coefficients of the desired linear form where substantial cancellations yield an additive approximation of $x_{j, s_{1}}$ or $x_{j, s_{2}}$.

Finally we combine all ingredients highlighted above to construct an anonymous game $\mathcal{G}_{\mathbf{A}}$ from polymatrix game A. This is done by first using the estimation lemma to compute, for each main $P_{i}$ coefficients of linear forms of probabilities $\operatorname{Pr}_{\mathcal{X}}\left[P_{i}, \mathbf{k}\right]$ seen by $P_{i}$ that yield additive approximations of $x_{j, s_{1}}$ and $x_{j, s_{2}}$. We then perturb payoff functions of players $P_{i}$ in the generalized radix game $\mathcal{G}_{n, N}^{*}$ using these coefficients so that 1 ) the resulting game $\mathcal{G}_{\mathbf{A}}$ is close to $\mathcal{G}_{n, N}^{*}$ and thus, any wellsupported equilibrium $\mathcal{X}$ of $\mathcal{G}_{\mathbf{A}}$ automatically satisfies the "scaling" property; 2) expected payoffs of $P_{i}$ playing $s_{1}, s_{2}$ in a well-supported equilibrium $\mathcal{X}$ of $\mathcal{G}_{\mathbf{A}}$ match additively expected payoffs of player $i$ playing rows $2 i-1,2 i$ in $\mathbf{A}$, given $\mathbf{y}$ derived from $\mathcal{X}$ by normalizing $\left(x_{j, s_{1}}, x_{j, s_{2}}\right)$ for each $j$. The correctness of the reduction, i.e., $\mathbf{y}$ is a $(1 / n)$-well-supported equilibrium of $\mathbf{A}$ whenever $\mathcal{X}$ is an $\epsilon$-well-supported equilibrium of $\mathcal{G}_{\mathbf{A}}$ with an exponentially small $\epsilon$, follows from these properties of $\mathcal{G}_{\mathrm{A}}$.

\subsection{Organization}

In Section 2, we define the radix game, and show that it has a unique Nash equilibrium as a warmup. We also use it to define the generalized radix game which serves as the base of our reduction. In section 3, we characterize well-supported Nash equilibria of anonymous games that are close to the generalized radix game (i.e., those that can be obtained by adding small perturbations to payoffs of the generalized radix game). In section 4, we prove the PPAD-hardness part of the main theorem. Our reduction relies on a crucial technical lemma, called the estimation lemma, which we prove in Section 5. We prove the membership in Section 6, and conclude with open problems in Section 7.

\section{Warm-up: Radix Game}

In this section, we first define a $(n+2)$-player anonymous game $\mathcal{G}_{n, N}$, called the radix game. As a warmup for the next section, we show that it has a unique Nash equilibrium. We then use the radix game to define the generalized radix game $\mathcal{G}_{n, N}^{*}$, by making a duplicate of a pure strategy in $\mathcal{G}_{n, N}$. The latter will serve as the base game for our polynomial-time reduction from polymatrix games. 


\subsection{Radix Game}

The radix game $\mathcal{G}_{n, N}$ to be defined has a unique Nash equilibrium of a specific form: given $N \geq 2$ as an integer parameter of the game, each of the $n$ "main" players mixes over the first two strategies with probabilities $1 / N^{i}$ and $1-1 / N^{i}$, respectively, for each $i \in[n]$, in the unique Nash equilibrium. The remaining two "special" players are created to achieve the aforementioned property.

Game 1 (Radix Game $\mathcal{G}_{n, N}$ ). Let $n \geq 1$ and $N \geq 2$ denote two integer parameters. Let $\delta=1 / N$.

Let $\mathcal{G}_{n, N}$ denote the following anonymous game with $n+2$ players $\left\{P_{1}, \ldots, P_{n}, Q, R\right\}$ and 6 pure strategies $\left\{s, t, q_{1}, q_{2}, r_{1}, r_{2}\right\}$. We refer to $\left\{P_{1}, \ldots, P_{n}\right\}$ as the main players. Each main player $P_{i}$ is only interested in strategies $s$ and $t$ (e.g., by setting her payoff of playing any other four actions to be -1 no matter what other players play). Player $Q$ is only interested in strategies $\left\{q_{1}, q_{2}\right\}$, and player $R$ is only interested in strategies $\left\{r_{1}, r_{2}\right\}$.

Next we define the payoff function of each player. When describing the payoff of a player below we always use $\mathbf{k}=\left(k_{s}, k_{t}, k_{q_{1}}, k_{q_{2}}, k_{r_{1}}, k_{r_{2}}\right)$ to denote the histogram of strategies this player sees.

1. For each $i \in[n]$, the payoff of player $P_{i}$ when she plays $s$ only depends on $k_{s}$ :

$$
\text { payoff }_{P_{i}}(s, \mathbf{k})= \begin{cases}\delta^{i}+\prod_{j \in[n]} \delta^{j} & \text { if } k_{s}=n-1 \\ \prod_{j \in[n]} \delta^{j} & \text { otherwise. }\end{cases}
$$

The payoff of player $P_{i}$ when she plays $t$ only depends on $k_{r_{1}}$ :

$$
\text { payoff }_{P_{i}}(t, \mathbf{k})= \begin{cases}2 & \text { if } k_{r_{1}}=1 \\ 0 & \text { otherwise }\end{cases}
$$

2. The payoff of player $Q$ when she plays $q_{1}$ or $q_{2}$ is given by

$$
\text { payoff }_{Q}\left(q_{1}, \mathbf{k}\right)=\left\{\begin{array}{ll}
1 & \text { if } k_{s}=n \\
0 & \text { otherwise }
\end{array} \quad \text { and } \quad \text { payoff }_{Q}\left(q_{2}, \mathbf{k}\right)= \begin{cases}1 & \text { if } k_{r_{1}}=1 \\
0 & \text { otherwise }\end{cases}\right.
$$

3. The payoff of player $R$ when she plays $r_{1}$ or $r_{2}$ is given by

$$
\text { payoff }_{R}\left(r_{1}, \mathbf{k}\right)=\left\{\begin{array}{ll}
1 & \text { if } k_{q_{1}}=1 \\
0 & \text { otherwise }
\end{array} \quad \text { and } \quad \text { payoff }_{R}\left(r_{2}, \mathbf{k}\right)= \begin{cases}1 & \text { if } k_{q_{2}}=1 \\
0 & \text { otherwise }\end{cases}\right.
$$

This finishes the definition of the radix game $\mathcal{G}_{n, N}$.

Fact 5. $\mathcal{G}_{n, N}$ is an anonymous game with payoff functions taking values from $[-1,2]$.

Since the main players $P_{i}$ are only interested in $\{s, t\}, Q$ is only interested in $\left\{q_{1}, q_{2}\right\}$, and $R$ is only interested in $\left\{r_{1}, r_{2}\right\}$, each Nash equilibrium $\mathcal{X}$ of $\mathcal{G}_{n, N}$ can be fully specified by a $(n+2)$-tuple $\mathcal{X}=\left(x_{1}, \ldots, x_{n}, y, z\right) \in[0,1]^{n+2}$, where $x_{i}$ denotes the probability of $P_{i}$ playing strategy $s$ for each $i \in[n], y$ denotes the probability of $Q$ playing $q_{1}$, and $z$ denotes the probability of $R$ playing $r_{1}$.

Given $\mathcal{X}=\left(x_{1}, \ldots, x_{n}, y, z\right)$ we calculate the expected payoff of each player as follows (we skip $\mathcal{X}$ in the expected payoffs $u_{p}(b, \mathcal{X})$, when $\mathcal{X}$ is clear from the context, and we use $u_{i}$ to denote the expected payoff of $P_{i}$ instead of $u_{P_{i}}$ for convenience): 
Fact 6. Given $\mathcal{X}=\left(x_{1}, \ldots, x_{n}, y, z\right)$, the expected payoff of player $P_{i}$ for playing $s$ is

$$
u_{i}(s)=\delta^{i} \cdot \operatorname{Pr}\left[k_{s}=n-1\right]+\prod_{j \in[n]} \delta^{j}=\delta^{i} \prod_{j \neq i \in[n]} x_{j}+\prod_{j \in[n]} \delta^{j} .
$$

The expected payoff of $P_{i}$ for playing $t$ is $u_{i}(t)=2 z$.

The expected payoff of player $Q$ for playing $q_{1}$ is

$$
u_{Q}\left(q_{1}\right)=\operatorname{Pr}\left[k_{s}=n\right]=\prod_{i \in[n]} x_{i}
$$

The expected payoff of $Q$ for playing $q_{2}$ is $u_{Q}\left(q_{2}\right)=z$.

The expected payoff of $R$ for playing $r_{1}$ is $u_{R}\left(r_{1}\right)=y$ and that for $r_{2}$ is $u_{R}\left(r_{2}\right)=1-y$.

We show that $x_{i}=\delta^{i}$ in a Nash equilibrium $\mathcal{X}$ of $\mathcal{G}_{n, N}$. We start with the following lemma.

Lemma 7. In a Nash equilibrium $\mathcal{X}=\left(x_{1}, \ldots, x_{n}, y, z\right)$ of $\mathcal{G}_{n, N}$, we have that $z=\prod_{i \in[n]} x_{i}$.

Proof. Assume for contradiction that $z>\prod_{i} x_{i}$. As $u_{Q}\left(q_{2}\right)>u_{Q}\left(q_{1}\right)$ and $\mathcal{X}$ is a Nash equilibrium, player $Q$ never plays $q_{1}$ and thus, $y=0$. This in turn implies $u_{R}\left(r_{2}\right)=1>0=u_{R}\left(r_{1}\right)$ and $z=0$, which contradicts with the assumption that $z>\prod_{i} x_{i} \geq 0$.

Next, assume for contradiction that $z<\prod_{i} x_{i}$, giving us that $u_{Q}\left(q_{2}\right)<u_{Q}\left(q_{1}\right)$. Player $Q$ never plays $q_{2}$ and $y=1$. This implies that $u_{R}\left(r_{1}\right)>u_{R}\left(r_{2}\right)$ and thus $z=1$, which contradicts with the assumption that $z<\prod_{i} x_{i} \leq 1$ (as $x_{i} \in[0,1]$ ). This finishes the proof of the lemma.

We now show that the radix game $\mathcal{G}_{n, N}$ has a unique Nash equilibrium $\mathcal{X}$ with $x_{i}=\delta^{i}$.

Lemma 8. In a Nash equilibrium $\mathcal{X}=\left(x_{1}, \ldots, x_{n}, y, z\right)$ of $\mathcal{G}_{n, N}$, we have $x_{i}=\delta^{i}$ for all $i \in[n]$.

Proof. First we show that $\prod_{i \in[n]} x_{i}=\prod_{i \in[n]} \delta^{i}$. Consider for contradiction the following two cases:

Case 1: $\prod_{i \in[n]} x_{i}<\prod_{i \in[n]} \delta^{i}$. Then there is an $i \in[n]$ such that $x_{i}<\delta^{i}$. For $P_{i}$, we have

$$
u_{i}(s)=\delta^{i} \prod_{j \neq i} x_{j}+\prod_{j \in[n]} \delta^{j}>\prod_{j \in[n]} x_{j}+\prod_{j \in[n]} x_{j}=2 \prod_{j \in[n]} x_{j}=2 z=u_{i}(t) .
$$

This implies that $x_{i}=1$, contradicting with the assumption that $x_{i}<\delta^{i}<1$ as $N \geq 2$.

Case 2: $\prod_{i \in[n]} x_{i}>\prod_{i \in[n]} \delta^{i}$. Then there is an $i \in[n]$ such that $x_{i}>\delta^{i}$. For $P_{i}$, we have

$$
u_{i}(s)=\delta^{i} \prod_{j \neq i} x_{j}+\prod_{j \in[n]} \delta^{j}<\prod_{j \in[n]} x_{j}+\prod_{j \in[n]} x_{j}=2 \prod_{j \in[n]} x_{j}=2 z=u_{i}(t) .
$$

This implies that $x_{i}=0$, contradicting with the assumption that $x_{i}>\delta^{i}>0$.

As a result, we must have $\prod_{i} x_{i}=\prod_{i} \delta^{i}$, which also implies that $x_{i}>0$ for all $i \in[n]$.

Now we show that $x_{i}=\delta^{i}$ for all $i$. Assume for contradiction that $x_{i} \neq \delta^{i}$ for some $i \in[n]$.

Case 1: $x_{i}<\delta^{i}$. Then the same strict inequality (2) holds for $P_{i}$, which implies that $x_{i}=1$, contradicting with the assumption that $x_{i}<\delta^{i}<1$ as $N \geq 2$. 
Case 2: $x_{i}>\delta^{i}$. Then the same strict inequality (3) holds for $P_{i}$, which implies that $x_{i}=0$, contradicting with the assumption that $x_{i}>\delta^{i}>0$.

This finishes the proof of the lemma.

Notice that Lemma 7 and 8 together imply that $\mathcal{G}_{n, N}$ has a unique Nash equilibrium because of Lemma 7 as well as the fact that $0<z<1$ implies $u_{R}\left(r_{1}\right)=y=1-y=u_{R}\left(r_{2}\right)$ and thus $y=1 / 2$.

\subsection{Generalized Radix Game}

We use $\mathcal{G}_{n, N}$ to define an anonymous game $\mathcal{G}_{n, N}^{*}$, called the generalized radix game, with the same set of $n+2$ players $\left\{P_{1}, \ldots, P_{n}, Q, R\right\}$ but seven strategies $\left\{s_{1}, s_{2}, t, q_{1}, q_{2}, r_{1}, r_{2}\right\}$. To this end, we replace strategy $s$ in $\mathcal{G}_{n, N}$ with two of its duplicate strategies $s_{1}, s_{2}$ in $\mathcal{G}_{n, N}^{*}$ and make sure that the players in $\mathcal{G}_{n, N}^{*}$ treat both $s_{1}$ and $s_{2}$ the same as the old strategy $s$, and have their payoff functions derived from those of players in $\mathcal{G}_{n, N}$ in this fashion. We will show in the next section that in any Nash equilibrium of $\mathcal{G}_{n, N}^{*}$, player $P_{i}$ must have probability exactly $\delta^{i}$ distributed among $s_{1}, s_{2}$.

For readers who are familiar with previous PPAD-hardness results of Nash equilibria in normal form games [DGP09, CDT09], this is the same trick used to derive the game generalized matching pennies from matching pennies. We define $\mathcal{G}_{n, N}^{*}$ formally as follows.

Game 2 (Generalized Radix Game $\mathcal{G}_{n, N}^{*}$ ). Let $n \geq 1$ and $N \geq 2$ be two parameters. Let $\delta=1 / N$. We use $\mathcal{G}_{n, N}^{*}$ to denote an anonymous game with the same $n+2$ players $\left\{P_{1}, \ldots, P_{n}, Q, R\right\}$ as $\mathcal{G}_{n, N}$ but now 7 pure strategies $\left\{s_{1}, s_{2}, t, q_{1}, q_{2}, r_{1}, r_{2}\right\}$. The payoff function payoff $T_{T}^{*}$ of a player $T$ in $\mathcal{G}_{n . N}^{*}$ is defined using payoff $T$ of the same player $T$ in $\mathcal{G}_{n, N}$ as follows:

$$
\operatorname{payoff}_{T}^{*}\left(b,\left(k_{s_{1}}, k_{s_{2}}, k_{t}, k_{q_{1}}, k_{q_{2}}, k_{r_{1}}, k_{r_{2}}\right)\right)=\operatorname{payoff}_{T}\left(\phi(b),\left(k_{s_{1}}+k_{s_{2}}, k_{t}, k_{q_{1}}, k_{q_{2}}, k_{r_{1}}, k_{r_{2}}\right)\right),
$$

where $\phi\left(s_{1}\right)=\phi\left(s_{2}\right)=s$ and $\phi(b)=b$ for every other pure strategy.

Since the payoff of player $P_{i}$ is always -1 when playing $q_{1}, q_{2}, r_{1}$ or $r_{2}$, she is only interested in $s_{1}, s_{2}$ and $t$. Similarly $Q$ is only interested in $q_{1}, q_{2}$ and $R$ is only interested in $r_{1}, r_{2}$. As a result, a Nash equilibrium $\mathcal{X}$ of $\mathcal{G}_{n, N}^{*}$ can be fully specified by $2 n+2$ numbers $\left(x_{i, 1}, x_{i, 2}, y, z: i \in[n]\right)$, where $x_{i, 1}$ (or $x_{i, 2}$ ) denotes the probability of $P_{i}$ playing strategy $s_{1}$ (or strategy $s_{2}$, respectively), so the probability of $P_{i}$ playing $t$ is $1-x_{i, 1}-x_{i, 2}$. We also let $x_{i}=x_{i, 1}+x_{i, 2}$ for each $i \in[n]$.

Given the definition of $\mathcal{G}_{n, N}^{*}$ from $\mathcal{G}_{n, N}$, Lemma 8 suggests $x_{i}=x_{i, 1}+x_{i, 2}=\delta^{i}$, for all $i \in[n]$, in every Nash equilibrium $\mathcal{X}$ of $\mathcal{G}_{n, N}^{*}$. This indeed follows from the main lemma of the next section concerning $\epsilon$-well-supported Nash equilibria of not only the generalized radix game $\mathcal{G}_{n, N}^{*}$ itself, but also anonymous games obtained by perturbing payoff functions of $\mathcal{G}_{n, N}^{*}$.

\section{Generalized Radix Game after Perturbation}

In this section, we analyze $\epsilon$-well-supported Nash equilibria of anonymous games obtained by perturbing payoff functions of the generalized radix game $\mathcal{G}_{n, N}^{*}$. Recall that $n \geq 1$ and $N \geq 2$, and we use payoff* to denote the payoff function of a player $T$ in $\mathcal{G}_{n, N}^{*}$. Given $x, y \in \mathbb{R}$ and $\xi \geq 0$, we write $x=y \pm \xi$ to denote $|x-y| \leq \xi$. We first define anonymous games that are close to $\mathcal{G}_{n, N}^{*}$.

Definition 9. For $\xi \geq 0$, we say an anonymous game $\mathcal{G}$ is $\xi$-close to $\mathcal{G}_{n, N}^{*}$ if 
1. $\mathcal{G}$ has the same set $\left\{P_{1}, \ldots, P_{n}, Q, R\right\}$ of players and same set of 7 strategies as $\mathcal{G}_{n, N}^{*}$.

2. For each player $T \in\left\{P_{1}, \ldots, P_{n}, Q, R\right\}$, her payoff function payoff $_{T}$ in $\mathcal{G}$ satisfies

$$
\operatorname{payoff}_{T}(b, \mathbf{k})=\operatorname{payoff}_{T}^{*}(b, \mathbf{k}) \pm \xi
$$

for all $b \in\left\{s_{1}, s_{2}, t, q_{1}, q_{2}, r_{1}, r_{2}\right\}$ and all histograms $\mathbf{k}$ of strategies played by $n+1$ players.

To characterize $\epsilon$-well-supported Nash equilibria of a game $\mathcal{G} \xi$-close to $\mathcal{G}_{n, N}^{*}$ we first show that when $\epsilon, \xi$ are small enough, each player in $\mathcal{G}$ remains only interested in a subset of strategies, i.e., $\left\{s_{1}, s_{2}, t\right\}$ for $P_{i},\left\{q_{1}, q_{2}\right\}$ for $Q$, and $\left\{r_{1}, r_{2}\right\}$ for $R$, in any $\epsilon$-well-supported Nash equilibrium of $\mathcal{G}$.

Lemma 10. Let $\mathcal{G}$ be an anonymous game $\xi$-close to $\mathcal{G}_{n, N}^{*}$ for some $\xi \geq 0$. When $2 \xi+\epsilon<1$, every $\epsilon$-well-supported Nash equilibrium of $\mathcal{G}$ satisfies: player $P_{i}$ only plays $\left\{s_{1}, s_{2}, t\right\}$; player $Q$ only plays $\left\{q_{1}, q_{2}\right\}$; player $R$ only plays $\left\{r_{1}, r_{2}\right\}$.

Proof. We only prove (1) since the proof of (2) and (3) is similar.

Given an $\epsilon$-well-supported Nash equilibrium $\mathcal{X}$, as the payoff of $P_{i}$ when playing $b \notin\left\{s_{1}, s_{2}, t\right\}$ is always -1 in $\mathcal{G}_{n, N}^{*}$, her expected payoff when playing $b$ in $\mathcal{G}$ is at most $-1+\xi$; as the payoff of $P_{i}$ when playing $b \in\left\{s_{1}, s_{2}, t\right\}$ is always nonnegative in $\mathcal{G}_{n, N}^{*}$, her expected payoff in $\mathcal{G}$ is at least $-\xi$. It follows from $2 \xi+\epsilon<1$ and the assumption of $\mathcal{X}$ being an $\epsilon$-well-supported equilibrium that $P_{i}$ only plays strategies in $\left\{s_{1}, s_{2}, t\right\}$ with positive probability.

It follows from Lemma 10 that an $\epsilon$-well-supported Nash equilibrium of $\mathcal{G}$ can be fully described by a tuple of $2 n+2$ numbers $\left(x_{i, 1}, x_{i, 2}, y, z: i \in[n]\right)$, when $\xi, \epsilon$ satisfy $2 \xi+\epsilon<1: x_{i, 1}$ denotes the probability of $P_{i}$ playing $s_{1}, x_{i, 2}$ denotes the probability of $P_{i}$ playing $s_{2}, y$ denotes the probability of $Q$ playing $q_{1}$, and $z$ denotes the probability of $R$ playing $r_{1}$.

Recall that $\delta=1 / N \leq 1 / 2$. Let $\kappa=\prod_{i \in[n]} \delta^{i}$. We prove the main lemma of this section.

Lemma 11. Let $\mathcal{G}$ denote an anonymous game that is $\xi$-close to $\mathcal{G}_{n, N}^{*}$. Suppose that $\xi, \epsilon \geq 0$ satisfy

$$
\tau=\frac{36 \xi+18 \epsilon}{\kappa} \leq 1 / 2
$$

Then every $\epsilon$-well-supported Nash equilibrium of $\mathcal{G}$ satisfies $x_{i, 1}+x_{i, 2}=\delta^{i} \pm \tau \delta^{i}$ for all $i \in[n]$.

Proof. Let $\mathcal{X}=\left(x_{i, 1}, x_{i, 2}, y, z: i \in[n]\right)$ be an $\epsilon$-well-supported Nash equilibrium of $\mathcal{G}$. For each $i \in$ $[n]$ we let $x_{i}=x_{i, 1}+x_{i, 2}$. Since $\mathcal{G}$ is $\xi$-close to $\mathcal{G}_{n, N}^{*}$, we have the following estimates:

1. The expected payoff of $P_{i}$ for playing strategy $s_{1}$ or $s_{2}$ is

$$
u_{i}\left(s_{1}\right), u_{i}\left(s_{2}\right)=\left(\delta^{i} \cdot \operatorname{Pr}\left[k_{s_{1}}+k_{s_{2}}=n-1\right]+\prod_{j \in[n]} \delta^{j}\right) \pm \xi=\left(\delta^{i} \prod_{j \neq i} x_{j}+\kappa\right) \pm \xi,
$$

where we write $k_{s_{1}}, k_{s_{2}}$ to denote the numbers of players that play $s_{1}, s_{2}$ respectively, as seen by player $P_{i}$ (same below). The expected payoff of $P_{i}$ for playing $t$ is $u_{i}(t)=2 z \pm \xi$.

2. The expected payoff of $Q$ for playing $q_{1}$ is

$$
u_{Q}\left(q_{1}\right)=\operatorname{Pr}\left[k_{s_{1}}+k_{s_{2}}=n\right] \pm \xi=\prod_{j \in[n]} x_{j} \pm \xi
$$

The expected payoff of $Q$ for playing $q_{2}$ is $u_{Q}\left(q_{2}\right)=z \pm \xi$. 
3. The expected payoff of $R$ for playing $r_{1}$ is $u_{R}\left(r_{1}\right)=y \pm \xi$ and for $r_{2}$ is $u_{R}\left(r_{2}\right)=(1-y) \pm \xi$.

To rest of the proof follows those of Lemma 7 and Lemma 8. First we show that $z$ must satisfy

$$
z=\prod_{j \in[n]} x_{j} \pm(2 \xi+\epsilon)
$$

The proof is the same as that of Lemma 7, using the assumption that $\mathcal{X}$ is $\epsilon$-well-supported.

Given (5), next we show that the $x_{i}$ 's satisfy

$$
\prod_{i \in[n]} x_{i}=\prod_{i \in[n]} \delta^{i} \pm(6 \xi+3 \epsilon)=\kappa \pm(6 \xi+3 \epsilon) .
$$

To this end we follow the proof of the first part of Lemma 8 and consider the following two cases:

Case 1: $\prod_{i \in[n]} x_{i}<\kappa-(6 \xi+3 \epsilon)$. Then there exists an $i \in[n]$ such that $x_{i}<\delta^{i}$. For $P_{i}$ :

$$
u_{i}\left(s_{1}\right) \geq \delta^{i} \prod_{j \neq i} x_{j}+\kappa-\xi>2 \prod_{j \in[n]} x_{j}+5 \xi+3 \epsilon \quad \text { and } \quad u_{i}(t) \leq 2 z+\xi \leq 2 \prod_{j \in[n]} x_{j}+5 \xi+2 \epsilon .
$$

This implies that $P_{i}$ does not play $t$ in $\mathcal{X}$, an $\epsilon$-well-supported Nash equilibrium of $\mathcal{G}$, and thus, $x_{i}=x_{i, 1}+x_{i, 2}=1$, contradicting with $x_{i}<\delta^{i}<1$ as $N \geq 2$.

Case 2: $\prod_{i \in[n]} x_{i}>\kappa+(6 \xi+3 \epsilon)$. Then there exists an $i \in[n]$ such that $x_{i}>\delta^{i}$. For $P_{i}$ :

$$
u_{i}\left(s_{1}\right), u_{i}\left(s_{2}\right) \leq \delta^{i} \prod_{j \neq i} x_{j}+\kappa+\xi<2 \prod_{j \in[n]} x_{j}-5 \xi-3 \epsilon \quad \text { and } \quad u_{i}(t) \geq 2 \prod_{j \in[n]} x_{j}-5 \xi-2 \epsilon .
$$

This implies that $P_{i}$ plays neither $s_{1}$ nor $s_{2}$ and thus, we have $x_{i, 1}=x_{i, 2}=0$ and $x_{i}=0$ as well, contradicting with $x_{i}>\delta^{i}>0$.

By (5) and (6), $z=\kappa \pm(8 \xi+4 \epsilon)$. (6) also implies that $x_{i}>0$ since $\kappa>0$ and $\kappa \geq 72 \xi+36 \epsilon$ by (4). Finally, assume for contradiction that either $x_{i}<(1-\tau) \delta^{i}$ or $x_{i}>(1+\tau) \delta^{i}$ for some $i \in[n]$.

Case 1: $x_{i}<(1-\tau) \delta^{i}$. Then using $\tau \leq 1 / 2$ and $1 \leq 1 /(1-\tau) \leq 2$, we have

$$
u_{i}\left(s_{1}\right)-u_{i}(t) \geq \delta^{i} \prod_{j \neq i} x_{j}+\kappa-2 z-2 \xi>\frac{\kappa-6 \xi-3 \epsilon}{1-\tau}+\kappa-2 z-2 \xi \geq \tau \kappa-30 \xi-14 \epsilon .
$$

Plugging in the definition of $\tau$ in (4), we have $u_{i}\left(s_{1}\right)-u_{i}(t)>\epsilon$ and thus, $x_{i}=1$, which contradicts with the assumption that $x_{i}<(1-\tau) \delta^{i}<1$.

Case 2: $x_{i}>(1+\tau) \delta^{i}$. Then using $\tau \leq 1 / 2$ and $2 / 3 \leq 1 /(1+\tau) \leq 1$, we have

$$
u_{i}\left(s_{1}\right)-u_{i}(t) \leq \delta^{i} \prod_{j \neq i} x_{j}+\kappa-2 z+2 \xi<\frac{\kappa+6 \xi+3 \epsilon}{1+\tau}+\kappa-2 z+2 \xi \leq-\frac{2 \tau \kappa}{3}+24 \xi+11 \epsilon .
$$

The same inequality holds for $u_{i}\left(s_{2}\right)-u_{i}(t)$. Plugging in (4), we have $u_{i}\left(s_{1}\right)-u_{i}(t)<-\epsilon$ as well as $u_{i}\left(s_{2}\right)-u_{i}(t)<-\epsilon$. This in turn implies that $x_{i, 1}=x_{i, 2}=0$ and thus, $x_{i}=0$, which contradicts with the assumption that $x_{i}>(1+\tau) \delta^{i}>0$.

This finishes the proof of the lemma. 


\section{Reduction from Polymatrix Games to Anonymous Games}

In this section we prove the hardness part of Theorem 1. For this purpose we present a polynomial time reduction from the problem of finding a $1 / n$-well-supported Nash equilibrium in a polymatrix game to the problem of finding an $\epsilon$-well-supported Nash equilibrium in an anonymous game with 7 strategies, for some exponentially small $\epsilon$. We first give some intuition behind this quite involved reduction in Section 4.1. Details of the reduction and the proof of its correctness are then presented in Section 4.2 and 4.3, respectively, with a key technical lemma proved in Section 5. We finish the proof of the hardness part in Section 4.4 by showing that any approximate Nash equilibrium of an anonymous game can be converted into a well-supported equilibrium efficiently (since Theorem 1 is concerned with approximate Nash equilibria).

\subsection{Overview of the Reduction}

Given as input a polymatrix game specified by a matrix $\mathbf{A} \in[0,1]^{2 n \times 2 n}$, our goal is to construct in polynomial time an anonymous game $\mathcal{G}_{\mathbf{A}}$, and show that every $\epsilon$-well-supported Nash equilibrium of $\mathcal{G}_{\mathbf{A}}$, where $\epsilon=1 / 2^{n^{6}}$, can be used to recover a $(1 / n)$-well-supported equilibrium of $\mathbf{A}$ in polynomial time. Note that this is not exactly the PPAD-hardness result as claimed in Theorem 1 but we will fill in the gap in Section 4.4 with some standard arguments.

Given $\mathbf{A}$, we construct $\mathcal{G}_{\mathbf{A}}$ by perturbing payoff functions of the Generalized Radix game $\mathcal{G}_{n, N}^{*}$ with $N=2^{n}$, so that $\mathcal{G}_{\mathbf{A}}$ is $\xi$-close to $\mathcal{G}_{n, N}^{*}$ for some exponentially small $\xi>0$ to be specified later. (Thus, $\mathcal{G}_{\mathbf{A}}$ has the same set of $n+2$ players $\left\{P_{1}, \ldots, P_{n}, Q, R\right\}$ as well as the same set of 7 strategies $\left\{s_{1}, s_{2}, t, q_{1}, q_{2}, r_{1}, r_{2}\right\}$ as $\mathcal{G}_{n, N}^{*}$.) By Lemma 10 and Lemma 11 we know that every $\epsilon$-well-supported equilibrium of $\mathcal{G}_{\mathbf{A}}$ can be fully described by a tuple $\mathcal{X}=\left(x_{i, 1}, x_{i, 2}, y, z: i \in[n]\right)$ that satisfies

$$
x_{i, 1}+x_{i, 2} \approx \delta^{i}
$$

for each $i \in[n]$, where $\delta=1 / N=1 / 2^{n}$.

Our construction of $\mathcal{G}_{\mathbf{A}}$ has player $P_{\ell}$ simulate row $2 \ell-1$ and $2 \ell$ of the polymatrix game $\mathbf{A}$ for each $\ell \in[n]$. The goal is to show at the end that, after normalizing $\left(x_{\ell, 1}, x_{\ell, 2}\right)$, i.e., probabilities of $P_{\ell}$ playing $s_{1}, s_{2}$ in an $\epsilon$-well-supported equilibrium $\mathcal{X}$ of $\mathcal{G}_{\mathbf{A}}$, into a distribution $\left(y_{2 \ell-1}, y_{2 \ell}\right)$ :

$$
y_{2 \ell-1}=\frac{x_{\ell, 1}}{x_{\ell, 1}+x_{\ell, 2}} \quad \text { and } \quad y_{2 \ell}=\frac{x_{\ell, 2}}{x_{\ell, 1}+x_{\ell, 2}}
$$

we get a $(1 / n)$-well-supported Nash equilibrium $\mathbf{y}=\left(y_{1}, \ldots, y_{2 n}\right)$ of $\mathbf{A}$. By $(7)$ we have

$$
y_{2 \ell-1} \approx N^{\ell} \cdot x_{\ell, 1} \text { and } y_{2 \ell} \approx N^{\ell} \cdot x_{\ell, 2} .
$$

For player $P_{\ell}$ to simulate row $2 \ell-1$ and $2 \ell$ of the polymatrix game $\mathbf{A}$, we perturb the original payoff function payoff* of $P_{\ell}$ in $\mathcal{G}_{n, N}^{*}$ in a way such that the following two linear forms of $\mathbf{y}$ :

$$
\mathbf{A}_{2 \ell-1} \cdot \mathbf{y}=\sum_{j \notin\{2 \ell-1,2 \ell\}} A_{2 \ell-1, j} \cdot y_{j} \quad \text { and } \quad \mathbf{A}_{2 \ell} \cdot \mathbf{y}=\sum_{j \notin\{2 \ell-1,2 \ell\}} A_{2 \ell, j} \cdot y_{j}
$$

appear as additive terms in the expected payoffs $u_{\ell}\left(s_{1}, \mathcal{X}\right)$ and $u_{\ell}\left(s_{2}, \mathcal{X}\right)$ of $P_{\ell}$ obtained from $s_{1}, s_{2}$, respectively. Let $u_{\ell}^{*}(\sigma, \mathcal{X})$ denote the expected payoff of player $P_{\ell}$ in the original generalized radix game $\mathcal{G}_{n, N}^{*}$ for strategies $\sigma \in\left\{s_{1}, s_{2}\right\}$. Then more specifically, we would like to perturb carefully the 
payoff functions of $\mathcal{G}_{n, N}^{*}$ such that for every $\ell \in[n]$, the expected payoffs of player $P_{\ell}$ in an $\epsilon$-wellsupported Nash equilibrium $\mathcal{X}$ of $\mathcal{G}_{\mathrm{A}}$ satisfy

$$
\begin{aligned}
u_{\ell}\left(s_{1}, \mathcal{X}\right) & \approx u_{\ell}^{*}\left(s_{1}, \mathcal{X}\right)+\xi^{*} \cdot \mathbf{A}_{2 \ell-1} \cdot \mathbf{y} \\
& \approx u_{\ell}^{*}\left(s_{1}, \mathcal{X}\right)+\xi^{*} \sum_{j \neq \ell} N^{j}\left(A_{2 \ell-1,2 j-1} \cdot x_{j, 1}+A_{2 \ell-1,2 j} \cdot x_{j, 2}\right) \\
u_{\ell}\left(s_{2}, \mathcal{X}\right) & \approx u_{\ell}^{*}\left(s_{2}, \mathcal{X}\right)+\xi^{*} \cdot \mathbf{A}_{2 \ell} \cdot \mathbf{y} \\
& \approx u_{\ell}^{*}\left(s_{2}, \mathcal{X}\right)+\xi^{*} \sum_{j \neq \ell} N^{j}\left(A_{2 \ell, 2 j-1} \cdot x_{j, 1}+A_{2 \ell, 2 j} \cdot x_{j, 2}\right)
\end{aligned}
$$

where $\xi^{*}$ is a parameter small enough to make sure that the resulting game is $\xi$-close to $\mathcal{G}_{n, N}^{*}$.

If one can perturb the payoff functions of players $P_{\ell}$ in $\mathcal{G}_{n, N}^{*}$ so that (9) and (10) hold for every $\epsilon$-well-supported Nash equilibrium $\mathcal{X}$ of $\mathcal{G}_{\mathbf{A}}$, then the vector $\mathbf{y}$ obtained from $\mathcal{X}$ using (8) must be a $(1 / n)$-well-supported equilibrium of $\mathbf{A}$. To see this, assume for contradiction that

$$
\mathbf{A}_{2 \ell-1} \cdot \mathbf{y}>\mathbf{A}_{2 \ell} \cdot \mathbf{y}+1 / n
$$

but $y_{2 \ell}>0$. Using (11), (9), and (10), we have $u_{\ell}\left(s_{1}, \mathcal{X}\right)$ is bigger than $u_{\ell}\left(s_{2}, \mathcal{X}\right)$ by $\xi^{*} / n$ (assuming that errors hidden in both (9) and (10) are negligible). As long as our choice of $\xi^{*}$ satisfies $\xi^{*} / n>\epsilon$ we must have $x_{\ell, 2}=0$ and thus, $y_{2 \ell}=0$ from (8).

However, perturbing the generalized radix game so that (9) and (10) hold is challenging. While

$$
\sum_{j \neq \ell} N^{j}\left(A_{2 \ell-1,2 j-1} \cdot x_{j, 1}+A_{2 \ell-1,2 j} \cdot x_{j, 2}\right) \quad \text { and } \sum_{j \neq \ell} N^{j}\left(A_{2 \ell, 2 j-1} \cdot x_{j, 1}+A_{2 \ell, 2 j} \cdot x_{j, 2}\right)
$$

are merely two linear forms of $\left(x_{j, 1}, x_{j, 2}: j \neq \ell\right)$ from $\mathcal{X}$, they are extremely difficult to obtain due to the nature of anonymous games: the expected payoff of player $P_{\ell}$ is

$$
u_{\ell}(\sigma, \mathcal{X})=\sum_{\mathbf{k} \in K} \operatorname{payoff}_{\ell}(\sigma, \mathbf{k}) \cdot \operatorname{Pr}_{\mathcal{X}}\left[P_{\ell}, \mathbf{k}\right]
$$

a linear form of $\operatorname{Pr}_{\mathcal{X}}\left[P_{\ell}, \mathbf{k}\right]$, the probability of $P_{\ell}$ seeing histogram $\mathbf{k}$ given $\mathcal{X}$. As each $\operatorname{Pr}_{\mathcal{X}}\left[P_{\ell}, \mathbf{k}\right]$ is a highly complex and symmetric expression of variables in $\mathcal{X}$, it is not clear how one can extract from (13) the desired linear forms of (12).

This is where the fact that $x_{i, 1}+x_{i, 2} \approx \delta^{i}$ helps us tremendously. (Recall that this holds as long as the generalized radix game $\mathcal{G}_{n, N}^{*}$ and $\mathcal{G}_{\mathbf{A}}$ are $\xi$-close.) The core of the construction of $\mathcal{G}_{\mathbf{A}}$ uses the following key technical lemma which we refer to as the estimation lemma. It shows that under any mixed strategy profile $\mathcal{X}=\left(x_{i, 1}, x_{i, 2}, y, z: i \in[n]\right)$ such that $x_{i, 1}+x_{i, 2} \approx \delta^{i}$, there is indeed a linear form of $\operatorname{Pr}_{\mathcal{X}}\left[P_{\ell}, \mathbf{k}\right]$ that gives us a close approximation of $x_{j, 1}$ (or $\left.x_{j, 2}\right), j \neq \ell$, and its coefficients can be computed in polynomial time in $n$. We delay its proof to Section 5 .

Lemma 12 (Estimation Lemma). Let $N=2^{n}$ and $\lambda=2^{-n^{3}}$. Given $\ell \in[n]$ and $j \neq \ell \in[n]$ one can compute in polynomial time in n vectors $\mathbf{B}^{[\ell, j]}, \mathbf{C}^{[\ell, j]}$ of length $|K|$ (indexed by $\mathbf{k} \in K$ ) such that every mixed strategy profile $\mathcal{X}=\left(x_{i, 1}, x_{i, 2}, y, z: i \in[n]\right)$ with $x_{i, 1}+x_{i, 2}=\delta^{i} \pm \lambda$ for all $i$ satisfies

$$
\sum_{\mathbf{k} \in K} B_{\mathbf{k}}^{[\ell, j]} \cdot \operatorname{Pr}_{\mathcal{X}}\left[P_{\ell}, \mathbf{k}\right]=x_{j, 1} \pm O\left(j^{2} \delta^{j+1}\right) \quad \text { and } \quad \sum_{\mathbf{k} \in K} C_{\mathbf{k}}^{[\ell, j]} \cdot \operatorname{Pr}_{\mathcal{X}}\left[P_{\ell}, \mathbf{k}\right]=x_{j, 2} \pm O\left(j^{2} \delta^{j+1}\right) .
$$

Moreover, the absolute value of each entry of $\mathbf{B}^{[\ell, j]}$ and $\mathbf{C}^{[\ell, j]}$ is at most $N^{n^{2}}$. 
With the estimation lemma in hand we can derive linear forms of $\operatorname{Pr}_{\mathcal{X}}\left[P_{\ell}, \mathbf{k}\right]$ that are close approximations of the two linear forms of $\left(x_{j, 1}, x_{j, 2}: j \neq \ell\right)$ in (12). We then use the coefficients of these linear forms of $\operatorname{Pr}_{\mathcal{X}}\left[P_{\ell}, \mathbf{k}\right]$ to perturb $\mathcal{G}_{n, N}^{*}$ and wrap up the construction of $\mathcal{G}_{\mathbf{A}}$.

\subsection{Construction of Anonymous Game $\mathcal{G}_{\mathrm{A}}$}

Let $\mathbf{A} \in[0,1]^{2 n \times 2 n}$ denote the input polymatrix game. We need the following parameters:

$$
N=2^{n}, \quad \delta=1 / N=2^{-n}, \quad \lambda=2^{-n^{3}}, \quad \xi=2^{-n^{4}}, \quad \xi^{*}=2^{-n^{5}} \text { and } \epsilon=2^{-n^{6}} .
$$

We remark that we do not attempt to optimize the parameters here but rather set them in different scales to facilitate the analysis later.

Game 3 (Construction of $\mathcal{G}_{\mathbf{A}}$ ). We use the polynomial-time algorithm promised in the Estimation Lemma to compute $\mathbf{B}^{[\ell, j]}$ and $\mathbf{C}^{[\ell, j]}$, for all $\ell \in[n]$ and $j \neq \ell \in[n]$.

Starting with the generalized radix game $\mathcal{G}_{n, N}^{*}$, we modify payoff functions of players $P_{1}, \ldots, P_{n}$ as follows (payoff functions of $Q$ and $R$ remain unchanged). Let payoff* denote the payoff function of $P_{\ell}$ in $\mathcal{G}_{n, N}^{*}$. Then for each player $P_{\ell}$ and each histogram $\mathbf{k} \in K$, we set

$$
\begin{aligned}
& \operatorname{payoff}_{\ell}\left(s_{1}, \mathbf{k}\right)=\operatorname{payoff}_{\ell}^{*}\left(s_{1}, \mathbf{k}\right)+\xi^{*} \sum_{j \neq \ell} N^{j}\left(A_{2 \ell-1,2 j-1} \cdot B_{\mathbf{k}}^{[\ell, j]}+A_{2 \ell-1,2 j} \cdot C_{\mathbf{k}}^{[\ell, j]}\right) \\
& \operatorname{payoff}_{\ell}\left(s_{2}, \mathbf{k}\right)=\operatorname{payoff}_{\ell}^{*}\left(s_{2}, \mathbf{k}\right)+\xi^{*} \sum_{j \neq \ell} N^{j}\left(A_{2 \ell, 2 j-1} \cdot B_{\mathbf{k}}^{[\ell, j]}+A_{2 \ell, 2 j} \cdot C_{\mathbf{k}}^{[\ell, j]}\right),
\end{aligned}
$$

and keep all other payoffs of $P_{\ell}$ the same (i.e., $\operatorname{payoff}_{\ell}(\sigma, \mathbf{k})=\operatorname{payoff}_{\ell}^{*}(\sigma, \mathbf{k})$ for all $\left.\sigma \notin\left\{s_{1}, s_{2}\right\}\right)$.

A few properties of $\mathcal{G}_{\mathbf{A}}$ then follow directly from its construction. First, observe that entries of $\mathbf{A}$ lie in $[0,1]$ and entries of $\mathbf{B}^{[\ell, j]}$ and $\mathbf{C}^{[\ell, j]}$ have absolute values at most $N^{n^{2}}=2^{n^{3}}$. We have

Property 13. Given $\mathbf{A} \in[0,1]^{2 n \times 2 n}, \mathcal{G}_{\mathbf{A}}$ is an anonymous game $\xi$-close to $\mathcal{G}_{n, N}^{*}$ where $\xi=2^{-n^{4}}$.

By Lemma 10, an $\epsilon$-well-supported Nash equilibrium of $\mathcal{G}_{\mathbf{A}}$ is fully described by a $(2 n+2)$-tuple $\mathcal{X}=\left(x_{i, 1}, x_{i, 2}, y, z: i \in[n]\right)$, where $P_{i}$ plays strategies $s_{1}, s_{2}$ and $t$ with probabilities $x_{i, 1}, x_{i, 2}$ and $1-x_{i, 1}-x_{i, 2}$, respectively. We also get the following corollary from Lemma 11.

Corollary 14. Every $\epsilon$-well-supported equilibrium $\mathcal{X}=\left(x_{i, 1}, x_{i, 2}, y, z: i \in[n]\right)$ of $\mathcal{G}_{\mathbf{A}}$ satisfies

$$
x_{i, 1}+x_{i, 2}=\delta^{i} \pm \lambda, \quad \text { for all } i \in[n] .
$$

Therefore, the conditions of the estimation lemma are met. It follows that

Property 15. Given an $\epsilon$-well-supported equilibrium $\mathcal{X}$ of $\mathcal{G}_{\mathbf{A}}$, the expected payoffs of $P_{\ell}$ satisfy

$$
\begin{aligned}
& u_{\ell}\left(s_{1}, \mathcal{X}\right)=u_{\ell}^{*}\left(s_{1}, \mathcal{X}\right)+\xi^{*} \sum_{j \neq \ell} N^{j}\left(A_{2 \ell-1,2 j-1} \cdot x_{j, 1}+A_{2 \ell-1,2 j} \cdot x_{j, 2}\right) \pm O\left(n^{3} \xi^{*} \delta\right) \quad \text { and } \\
& u_{\ell}\left(s_{2}, \mathcal{X}\right)=u_{\ell}^{*}\left(s_{2}, \mathcal{X}\right)+\xi^{*} \sum_{j \neq \ell} N^{j}\left(A_{2 \ell, 2 j-1} \cdot x_{j, 1}+A_{2 \ell, 2 j} \cdot x_{j, 2}\right) \pm O\left(n^{3} \xi^{*} \delta\right) .
\end{aligned}
$$




\subsection{Correctness of the Reduction}

We are now ready to show that, given an $\epsilon$-well-supported Nash equilibrium $\mathcal{X}$ of $\mathcal{G}_{\mathbf{A}}$, the vector $\mathbf{y}$ derived from $\mathcal{X}$ using $(8)$ is a $(1 / n)$-well-supported Nash equilibrium of the polymatrix game $\mathbf{A}$.

Lemma 16. Let $\mathcal{X}=\left(x_{i, 1}, x_{i, 2}, y, z: i \in[n]\right)$ be an $\epsilon$-well supported Nash equilibrium of $\mathcal{G}_{\mathbf{A}}$. Then the vector $\mathbf{y} \in[0,1]^{2 n}$ derived from $\mathcal{X}$ using $(8)$ is a $(1 / n)$-well-supported Nash equilibrium of $\mathbf{A}$.

Proof. Firstly, note that $x_{i, 1}+x_{i, 2}>0$ so $\mathbf{y}$ is well defined and satisfies $y_{2 i-1}+y_{2 i}=1$ for all $i$.

Assume towards a contradiction that $\mathbf{y}$ derived from $\mathcal{X}$ using (8) is not a $(1 / n)$-well-supported Nash equilibrium of $\mathbf{A}$, i.e., there is a player $\ell \in[n]$ such that, without loss of generality,

$$
\mathbf{A}_{2 \ell-1} \cdot \mathbf{y}>\mathbf{A}_{2 \ell} \cdot \mathbf{y}+1 / n
$$

but $y_{2 \ell}>0$, which in turn implies that $x_{\ell, 2}>0$.

Since $x_{j, 1}+x_{j, 2}=\delta^{j} \pm \lambda$, we have

$$
y_{2 j-1}=\frac{x_{j, 1}}{x_{j, 1}+x_{j, 2}}=N^{j} x_{j, 1} \pm O\left(N^{2 j} \lambda\right)=N^{j} x_{j, 1} \pm O\left(N^{2 n} \lambda\right) .
$$

Similarly we also have $y_{2 j}=N^{j} x_{j, 2} \pm O\left(N^{2 n} \lambda\right)$. Combining these with Property 15 , we have

$$
\begin{aligned}
& u_{\ell}\left(s_{1}, \mathcal{X}\right)=u_{\ell}^{*}\left(s_{1}, \mathcal{X}\right)+\xi^{*} \cdot \mathbf{A}_{2 \ell-1} \cdot \mathbf{y} \pm\left(O\left(n^{3} \xi^{*} \delta\right)+O\left(n \xi^{*} N^{2 n} \lambda\right)\right) \quad \text { and } \\
& u_{\ell}\left(s_{2}, \mathcal{X}\right)=u_{\ell}^{*}\left(s_{2}, \mathcal{X}\right)+\xi^{*} \cdot \mathbf{A}_{2 \ell} \cdot \mathbf{y} \pm\left(O\left(n^{3} \xi^{*} \delta\right)+O\left(n \xi^{*} N^{2 n} \lambda\right)\right) .
\end{aligned}
$$

By our choices of parameters, $n \xi^{*} N^{2 n} \lambda \ll n^{3} \xi^{*} \delta$ so the former can be absorbed into the latter.

Combining (14) and (15) (as well as the fact that $u_{\ell}^{*}\left(s_{1}, \mathcal{X}\right)=u_{\ell}^{*}\left(s_{2}, \mathcal{X}\right)$ because the payoffs of $s_{1}$ and $s_{2}$ are exactly the same in the generalized radix game $\left.\mathcal{G}_{n, N}^{*}\right)$, we have

$$
u_{\ell}\left(s_{1}, \mathcal{X}\right)-u_{\ell}\left(s_{2}, \mathcal{X}\right) \geq \xi^{*}\left(\mathbf{A}_{2 \ell-1} \cdot \mathbf{y}-\mathbf{A}_{2 \ell} \cdot \mathbf{y}\right)-O\left(n^{3} \xi^{*} \delta\right) \geq \xi^{*} / n-O\left(n^{3} \xi^{*} \delta\right)>\epsilon,
$$

for sufficiently large $n$, by our choices of parameters $\delta, \xi^{*}$ and $\epsilon$. It then follows that $x_{\ell, 2}=0$, since $\mathcal{X}$ is assumed to be an $\epsilon$-well-supported Nash equilibrium of $\mathcal{G}_{\mathbf{A}}$, contradicting with $y_{2 \ell}>0$. This finishes the proof.

\subsection{Proof of the Hardness Part of Theorem 1}

From our definitions of $\mathcal{G}_{n, N}^{*}$ and $\mathcal{G}_{\mathbf{A}}$, it is clear that all payoffs of $\mathcal{G}_{\mathbf{A}}$ are in [-1,3]. Using standard arguments (invariance of Nash equilibria under shifting and scaling), we can easily see that given an anonymous game $\mathcal{G}=\left(n, \alpha\right.$, payoff $\left.\left._{p}\right\}\right)$ such that all payoffs are in the interval $[a, b]$, where $a, b \in \mathbb{R}$ and $a<b$, a mixed strategy profile $\mathcal{X}$ is an $(b-a) \epsilon$-well-supported equilibrium of $\mathcal{G}$ if and only if $\mathcal{X}$ is an $\epsilon$-well-supported equilibrium of $\mathcal{G}^{\prime}=\left(n, \alpha,\left\{\right.\right.$ payoff $\left.\left._{p}^{\prime}\right\}\right)$, where

$$
\operatorname{payoff}_{p}^{\prime}(\sigma, \mathbf{k})=\frac{\text { payoff }_{p}(\sigma, \mathbf{k})-a}{b-a}
$$

The new game $\mathcal{G}^{\prime}$ now has all payoffs from in $[0,1]$.

As a result, we can construct $\mathcal{G}_{\mathbf{A}}^{\prime}$ from $\mathcal{G}_{\mathbf{A}}$ in polynomial time such that all payoffs of $\mathcal{G}_{\mathbf{A}}^{\prime}$ lie in $[0,1]$, and Lemma 16 holds for all $(\epsilon / 4)$-well-supported Nash equilibria of $\mathcal{G}_{\mathbf{A}}^{\prime}$. It follows that 
Corollary 17. Fix any $\alpha \geq 7$. The problem of finding a $2^{-\left(n^{6}+2\right)}$-well-supported Nash equilibrium of an anonymous game with $\alpha$ actions and $[0,1]$ payoffs is PPAD-hard.

This can be further strengthened using a standard padding argument.

Lemma 18. Fix any $\alpha \in \mathbb{N}$ and $a>b>0$. There is a polynomial-time reduction from the problem of finding a $\left(2^{-n^{a}}\right)$-well-supported equilibrium to that of finding a $\left(2^{-n^{b}}\right)$-well-supported equilibrium, in an anonymous game with $\alpha$ actions and $[0,1]$ payoffs.

Proof. For convenience, we will refer to the problem of finding a $\left(2^{-n^{a}}\right)$-well-supported equilibrium as problem $\mathrm{A}$ and the other as problem $\mathrm{B}$.

Let $\mathcal{G}=\left(n, \alpha,\left\{\right.\right.$ payoff $\left.\left._{p}\right\}\right)$ denote an input anonymous game of problem $\mathrm{A}$. We define a new game $\operatorname{pad} \mathcal{G}=\left(n^{t}, \alpha,\left\{\right.\right.$ payoff $\left.\left._{p}^{\prime}\right\}\right)$ as follows, where $t=a / b>1$ and thus, $n^{t}>n$. To this end, define a map $\phi: \mathbb{Z}^{\alpha} \rightarrow \mathbb{Z}^{\alpha}$ such that $\phi\left(k_{1}, \ldots, k_{\alpha}\right)=\left(k_{1}-\left(n^{t}-n\right), k_{2}, \ldots, k_{\alpha}\right)$. We then define payoff functions of players $\left\{1, \ldots, n^{t}\right\}$ in padG as follows:

- For each $i>n$, the payoff function of player $i$ is given by

$$
\text { payoff }_{i}^{\prime}(\sigma, \mathbf{k})= \begin{cases}1 & \text { if } \sigma=1 \\ 0 & \text { otherwise }\end{cases}
$$

So player $i$ always plays strategy 1 in any $\epsilon$-well-supported equilibrium with $\epsilon<1$.

- The payoff of each player $i \in[n]$ is given by

$$
\text { payoff }_{i}^{\prime}(\sigma, \mathbf{k})= \begin{cases}\operatorname{payoff}_{i}(\sigma, \phi(\mathbf{k})) & \text { if } k_{1} \geq n^{t}-n \\ 0 & \text { otherwise }\end{cases}
$$

Note that in any $\epsilon$-well-supported equilibrium with $\epsilon<1$, the latter case never occurs.

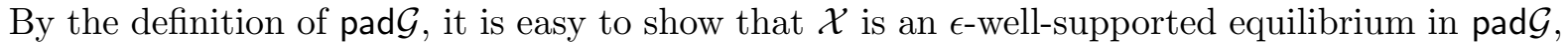
for some $\epsilon<1$, iff 1 ) each player $i>n$ plays strategy 1 with probability 1 and 2) the mixed strategy profile of the first $n$ players in $\mathcal{X}$ is an $\epsilon$-well-supported equilibrium of $\mathcal{G}$. As a result, a solution to padG as an input of problem B must be an $\epsilon$-approximate equilibrium of $\mathcal{G}$ with $\epsilon=2^{-\left(n^{t}\right)^{b}}=2^{-n^{a}}$. As pad $\mathcal{G}$ can be constructed from $\mathcal{G}$ in polynomial time, this finishes the proof of the lemma.

Combining Corollary 17 and Lemma 18, we have

Corollary 19. Fix any $\alpha \geq 7$ and $c>0$. The problem of finding a $\left(2^{-n^{c}}\right)$-well-supported Nash equilibrium in an anonymous game with $\alpha$ actions and $[0,1]$ payoffs is PPAD-hard.

To prove the hardness part of Theorem 1, we next give a polynomial-time algorithm to compute a well-supported equilibrium from an approximate equilibrium.

Lemma 20 (From Approximate to Well-Supported Nash Equilibria). Let $\mathcal{G}=(n, \alpha,\{$ payoff $p\})$ be an anonymous game with payoffs from $[0,1]$. Given an $\epsilon^{2} /(16 \alpha n)$-approximate Nash equilibrium $\mathcal{X}$ of $\mathcal{G}$, one can compute in polynomial time an $\epsilon$-well-supported Nash equilibrium $\mathcal{Y}$ of $\mathcal{G}$. 
Proof. Let $\mathcal{X}=\left(\mathbf{x}_{i}: i \in[n]\right)$ be an $\epsilon^{\prime}$-approximate Nash equilibrium of $\mathcal{G}$, with $\epsilon^{\prime}=\epsilon^{2} /(16 \alpha n)$. For each player $i \in[n]$, we have for any mixed strategy $\mathbf{x}_{i}^{\prime}$,

$$
u_{i}\left(\mathbf{x}_{i}^{\prime}, \mathcal{X}_{-i}\right) \leq u_{i}(\mathcal{X})+\epsilon^{\prime}
$$

where we let $u_{i}\left(\mathbf{x}_{i}^{\prime}, \mathcal{X}_{-i}\right)$ denote the expected payoff of player $i$ when she plays $\mathbf{x}_{i}^{\prime}$ and other players play $\mathcal{X}_{-i}$. Let $\sigma_{i}$ be a strategy with the highest expected payoff for player $i$ (with respect to $\mathcal{X}_{-i}$ ):

$$
u_{i}\left(\sigma_{i}, \mathcal{X}\right)=\max _{k \in[\alpha]} u_{i}(k, \mathcal{X})
$$

and let $J_{i}=\left\{j: u_{i}\left(\sigma_{i}, \mathcal{X}\right) \geq u_{i}(j, \mathcal{X})+\epsilon / 2\right\}$. We then define a mixed strategy $\mathbf{y}_{i}$ for player $i$ using $\mathbf{x}_{i}, \sigma_{i}$ and $J_{i}$ as follows: Set $y_{i, j}=0$ for all $j \in J_{i}$, and set

$$
y_{i, \sigma_{i}}=x_{i, \sigma_{i}}+\sum_{j \in J_{i}} x_{i, j}
$$

All other entries of $\mathbf{y}_{i}$ are the same as $\mathbf{x}_{i}$. As $\mathbf{y}_{i}$ increases the expected payoff of player $i$ by at least

$$
(\epsilon / 2) \cdot \sum_{j \in J_{i}} x_{i, j}
$$

we have from (17) that $\sum_{j \in J_{i}} x_{i, j} \leq 2 \epsilon^{\prime} / \epsilon$.

Repeating this for every player $i \in[n]$, we obtain a new mixed strategy profile $\mathcal{Y}$ (clearly $\mathcal{Y}$ can be computed in polynomial time given $\mathcal{X}$ ). We finish the proof of the lemma by showing that $\mathcal{Y}$ is indeed an $\epsilon$-well-supported Nash equilibrium of $\mathcal{G}$. Below we write $\zeta=2 \epsilon^{\prime} / \epsilon$.

First, by the definition of $\mathcal{Y},\left|x_{i, j}-y_{i, j}\right| \leq \zeta$ for all $i, j$. Thus, for any pure strategy profile $\mathbf{s}_{-i}$,

$$
\begin{aligned}
& \prod_{q \neq i} y_{q, s_{q}} \geq \prod_{q \neq i} \max \left\{0, x_{q, s_{q}}-\zeta\right\} \geq \prod_{q \neq i} x_{q, s_{q}}-\zeta \cdot \sum_{q \neq i} \prod_{p \notin\{i, q\}} x_{p, s_{p}} \quad \text { and } \\
& \prod_{q \neq i} x_{q, s_{q}} \geq \prod_{q \neq i} \max \left\{0, y_{q, s_{q}}-\zeta\right\} \geq \prod_{q \neq i} y_{q, s_{q}}-\zeta \cdot \sum_{q \neq i} \prod_{p \notin\{i, q\}} y_{p, s_{p}} .
\end{aligned}
$$

Since all payoffs are in $[0,1]$, we have for any player $i \in[n]$ and pure strategy $j \in[\alpha]$ that

$$
\begin{aligned}
\left|u_{i}(j, \mathcal{Y})-u_{i}(j, \mathcal{X})\right| & \leq \sum_{\mathbf{s}_{-i} \in[\alpha]^{n-1}}\left|\prod_{q \neq i} y_{q, s_{q}}-\prod_{q \neq i} x_{q, s_{q}}\right| \\
& \leq \zeta \sum_{\mathbf{s}_{-i}} \sum_{q \neq i} \prod_{p \notin\{i, q\}} x_{p, s_{p}}+\zeta \sum_{\mathbf{s}_{-i}} \sum_{q \neq i} \prod_{p \notin\{i, q\}} y_{p, s_{p}} \\
& =\zeta \sum_{q \neq i} \sum_{\mathbf{s}_{-i}} \prod_{p \notin\{i, q\}} x_{p, s_{p}}+\zeta \sum_{q \neq i} \sum_{\mathbf{s}_{-i}} \prod_{p \notin\{i, q\}} y_{p, s_{p}} \\
& \leq \alpha \zeta \sum_{q \neq i}\left(\sum_{s_{r}: r \notin\{i, q\}} \prod_{p \notin\{i, q\}} x_{p, s_{p}}\right)+\alpha \zeta \sum_{q \neq i}\left(\sum_{s_{r}: r \notin\{i, q\}} \prod_{p \notin\{i, q\}} y_{p, s_{p}}\right) \\
& =2(n-1) \alpha \zeta .
\end{aligned}
$$


This implies that for any pure strategies $j, k \in[\alpha]$ we have

$$
\left|\left(u_{i}(j, \mathcal{X})-u_{i}(k, \mathcal{X})\right)-\left(u_{i}(j, \mathcal{Y})-u_{i}(k, \mathcal{Y})\right)\right|<\epsilon / 2 .
$$

Therefore, the new mixed strategy profile $\mathcal{Y}=\left(\mathbf{y}_{i}: i \in[n]\right)$ satisfies

$$
u_{i}(j, \mathcal{Y})<u_{i}(k, \mathcal{Y})+\epsilon \Rightarrow u_{i}(j, \mathcal{X})<u_{i}(k, \mathcal{X})+\epsilon / 2 \Rightarrow y_{i, j}=0
$$

for all $i, j$ and $k$. This finishes the proof of the lemma.

Fix any $\alpha \geq 7$ and $c>0$. It then follows from Lemma 20 that the problem of finding a $\left(2^{-n^{c / 2}}\right)$ well-supported equilibrium in an anonymous game with $\alpha$ actions and [0,1] payoffs is polynomialtime reducible to problem $(\alpha, c)$-AnONYMOUS. As the former problem is PPAD-hard by Corollary $19,(\alpha, c)$-Anonymous is PPAD-hard. The finishes the proof of the hardness part of Theorem 1.

\section{Proof of the Estimation Lemma}

We prove the estimation lemma (Lemma 12) in this section.

Recall that there are $n$ main players $P_{1}, \ldots, P_{n}$, and they are only interested in three strategies $\left\{s_{1}, s_{2}, t\right\}$. For convenience we will refer to $s_{1}$ as strategy $1, s_{2}$ as strategy 2 , and $t$ as strategy 3 in this section. Player $P_{i}$ plays strategy $b \in[3]$ with probability $x_{i, b}$, and $\sum_{b} x_{i, b}=1$. While $x_{i, b}$ 's are unknown variables, by the assumption of the lemma we are guaranteed that

$$
x_{i, 1}+x_{i, 2}=\delta^{i} \pm \lambda, \quad \text { where } \lambda=\delta^{n^{2}} .
$$

Throughout this section we will fix two distinct integers $r, \ell \in[n]$, and the goal will be to derive an approximation of the unknown $x_{r, 1}$ for $P_{\ell}$ using a linear form of the following probabilities:

$$
\left\{\operatorname{Pr}\left[k_{1}=i, k_{2}=j\right]: i, j \in[0: n-1]\right\}, \quad \text { where } \operatorname{Pr}\left[k_{1}=i, k_{2}=j\right]=\sum_{\substack{\mathbf{k} \in K \\ k_{1}=i, k_{2}=j}} \operatorname{Pr}_{\mathcal{X}}\left[P_{\ell}, \mathbf{k}\right],
$$

and $k_{b}$ denotes the random variable that counts players playing $b \in[3]$ other than player $P_{\ell}$ herself. We will show that coefficients in the desired linear form can be computed in polynomial time in $n$.

First we would like to give the reader some intuition for the rest of the section, by showing how one can get a good estimate of $x_{1,1}$ and $x_{2,1}$, assuming $\ell>2$. We believe this to be useful for more easily understanding the rest of the section, but the reader should feel free to skip it, if desired.

Estimating $x_{1,1}$ and $x_{2,1}$ (Informal). As $N=2^{n}$ is large, we have $x_{i, 3} \approx 1$ for each $i$. This gives

$$
\operatorname{Pr}\left[k_{1}=1, k_{2}=0\right] \approx x_{1,1}+x_{2,1}+\cdots+x_{n, 1}=x_{1,1} \pm O\left(\delta^{2}\right)
$$

as $x_{i, 1} \leq \delta^{i}+\lambda$. Similarly, $\operatorname{Pr}\left[k_{1}=2, k_{2}=0\right] \approx x_{1,1} x_{2,1} \pm O\left(\delta^{4}\right)$. Using $x_{i, 1}+x_{i, 2} \approx \delta^{i}$, we have

$$
\operatorname{Pr}\left[k_{1}=k_{2}=1\right] \approx x_{1,1}\left(\delta^{2}-x_{2,1}\right)+x_{2,1}\left(\delta-x_{1,1}\right) \pm O\left(\delta^{4}\right)=\delta^{2} x_{1,1}+\delta x_{2,1}-2 x_{1,1} x_{2,1} \pm O\left(\delta^{4}\right) .
$$

Combining all three estimates, we have

$$
N\left(\operatorname{Pr}\left[k_{1}=k_{2}=1\right]+2 \cdot \operatorname{Pr}\left[k_{1}=2, k_{2}=0\right]\right)-\delta \cdot \operatorname{Pr}\left[k_{1}=1, k_{2}=0\right] \approx x_{2,1} \pm O\left(\delta^{3}\right) .
$$

Since $x_{2,1} \leq \delta^{2}+\lambda$, the linear form on the LHS gives us an additive approximation of $x_{2,1}$. 
We need some notation in order to generalize and formalize this. Let $\mathcal{S}=[n] \backslash\{\ell\}$, the set of players observed by player $P_{\ell}$. Let $k_{b}, b \in[3]$, denote the random variable that counts players from $\mathcal{S}$ that play strategy $b$. We write $\mathcal{L}=\{i \in \mathcal{S}: i \leq r\}$ and $m=|\mathcal{L}|$, i.e., $\mathcal{L}=[r]$ and $m=r$ if $\ell>r$, and $\mathcal{L}=[r] \backslash\{\ell\}$ and $m=r-1$ if $\ell<r$. We start by understanding the following probabilities

$$
\left\{\operatorname{Pr}\left[k_{1}=m-j, k_{2}=j\right]: j \in[0: m]\right\} .
$$

It will become clear that players from $\mathcal{S} \backslash \mathcal{L}$ have probabilities too small to significantly affect these probabilities (so their contribution will just be absorbed into the error term).

For $j \in[0: m]$, let $\Delta_{j}$ denote the set of partitions of $\mathcal{S}$ into sets of size $m-j, j$ and $n-1-m$ :

$\Delta_{j}=\left\{\left(\mathcal{S}_{1}, \mathcal{S}_{2}, \mathcal{S}_{3}\right): \mathcal{S}_{1}, \mathcal{S}_{2}, \mathcal{S}_{3}\right.$ are pairwise disjoint, $\left.\mathcal{S}_{1} \cup \mathcal{S}_{2} \cup \mathcal{S}_{3}=\mathcal{S},\left|\mathcal{S}_{1}\right|=m-j,\left|\mathcal{S}_{2}\right|=j\right\}$.

So, by definition, we have

$$
\operatorname{Pr}\left[k_{1}=m-j, k_{2}=j\right]=\sum_{\left(\mathcal{S}_{1}, \mathcal{S}_{2}, \mathcal{S}_{3}\right) \in \Delta_{j}}\left(\prod_{i \in \mathcal{S}_{1}} x_{i, 1} \prod_{i \in \mathcal{S}_{2}} x_{i, 2} \prod_{i \in \mathcal{S}_{3}} x_{i, 3}\right) .
$$

By (18) we can write $x_{i, 1}+x_{i, 2}=\delta^{i}+\lambda_{i}$ for some $\lambda_{i}$ with $\left|\lambda_{i}\right| \leq \lambda$. We can substitute to get

$$
\operatorname{Pr}\left[k_{1}=m-j, k_{2}=j\right]=\sum_{\left(\mathcal{S}_{1}, \mathcal{S}_{2}, \mathcal{S}_{3}\right) \in \Delta_{j}}\left(\prod_{i \in \mathcal{S}_{1}} x_{i, 1} \prod_{i \in \mathcal{S}_{2}}\left(\delta^{i}+\lambda_{i}-x_{i, 1}\right) \prod_{i \in \mathcal{S}_{3}}\left(1-\delta^{i}-\lambda_{i}\right)\right) .
$$

Next, we split $\Delta_{j}$ into two sets $\Delta_{j}^{*}$ and $\Delta_{j}^{\prime}:\left(\mathcal{S}_{1}, \mathcal{S}_{2}, \mathcal{S}_{3}\right) \in \Delta_{j}$ is in $\Delta_{j}^{*}$ if $\mathcal{S}_{1} \cup \mathcal{S}_{2}=\mathcal{L}$; otherwise, it is in $\Delta_{j}^{\prime}$. This splits the sum in (20) into two sums accordingly, one over $\Delta_{j}^{*}$ and one over $\Delta_{j}^{\prime}$. We show in the following lemma that the contribution from the second sum is negligible.

Lemma 21. Given the parameters in (18), we have

$$
\sum_{\left(\mathcal{S}_{1}, \mathcal{S}_{2}, \mathcal{S}_{3}\right) \in \Delta_{j}^{\prime}}\left(\prod_{i \in \mathcal{S}_{1}} x_{i, 1} \prod_{i \in \mathcal{S}_{2}}\left(\delta^{i}+\lambda_{i}-x_{i, 1}\right) \prod_{i \in \mathcal{S}_{3}}\left(1-\delta^{i}-\lambda_{i}\right)\right)=O\left(\delta \prod_{i \in \mathcal{L}} \delta^{i}\right) .
$$

Proof. Since all terms in the sum are nonnegative, it suffices to show that

$$
\sum_{\left(\mathcal{S}_{1}, \mathcal{S}_{2}, \mathcal{S}_{3}\right) \in \Delta_{j}^{\prime}}\left(\prod_{i \in \mathcal{S}_{1}} x_{i, 1} \prod_{i \in \mathcal{S}_{2}}\left(\delta^{i}+\lambda_{i}-x_{i, 1}\right)\right)=O\left(\delta \prod_{i \in \mathcal{L}} \delta^{i}\right) .
$$

Fix a set $\mathcal{T} \subseteq \mathcal{S}$ such that $|\mathcal{T}|=m$ but $\mathcal{T} \neq \mathcal{L}$. We have

$$
\prod_{i \in \mathcal{T}}\left(\delta^{i}+\lambda_{i}\right)=\prod_{i \in \mathcal{T}}\left(x_{i, 1}+\left(\delta^{i}+\lambda_{i}-x_{i, 1}\right)\right)=\sum_{\mathcal{S}_{1} \subseteq \mathcal{T}}\left(\prod_{i \in \mathcal{S}_{1}} x_{i, 1} \prod_{i \in \mathcal{T} \backslash \mathcal{S}_{1}}\left(\delta^{i}+\lambda_{i}-x_{i, 1}\right)\right) .
$$

Since every term on the RHS is nonnegative, we have

$$
\sum_{\substack{\mathcal{S}_{1} \subseteq \mathcal{T} \\\left|\mathcal{S}_{1}\right|=m-j}}\left(\prod_{i \in \mathcal{S}_{1}} x_{i, 1} \prod_{i \in \mathcal{T} \backslash \mathcal{S}_{1}}\left(\delta^{i}+\lambda_{i}-x_{i, 1}\right)\right) \leq \prod_{i \in \mathcal{T}}\left(\delta^{i}+\lambda_{i}\right)=(1+o(1)) \cdot \prod_{i \in \mathcal{T}} \delta^{i},
$$


given that $\lambda_{i}=\delta^{n^{2}}$ in $(18)$. Let $h(\mathcal{T})=\prod_{i \in \mathcal{T}} \delta^{i}$. To prove $(21)$, it now suffices to show that

$$
\sum_{\substack{\mathcal{T} \subseteq \mathcal{S} \\|\mathcal{T}|=m, \mathcal{T} \neq \mathcal{L}}} h(\mathcal{T})=O(\delta \cdot h(\mathcal{L}))=O\left(\delta \prod_{i \in \mathcal{L}} \delta^{i}\right)
$$

For this purpose, notice that $h(\mathcal{T}) \leq \delta \cdot h(\mathcal{L})$ for any $\mathcal{T}$ such that $\mathcal{T} \subseteq \mathcal{S},|\mathcal{T}|=m$, but $\mathcal{T} \neq \mathcal{L}$. It is also easy to see that there is at most one $\mathcal{T}$ such that $h(\mathcal{T})=\delta \cdot h(\mathcal{L})$. Because every other $\mathcal{T}$ has $h(\mathcal{T}) \leq \delta^{2} \cdot h(\mathcal{L})$ and the total number of $\mathcal{T}$ 's is at most $2^{n-1}=N / 2$, we have

$$
\sum_{\mathcal{T}} h(\mathcal{T}) \leq \delta \cdot h(\mathcal{L})+(N / 2) \cdot \delta^{2} \cdot h(\mathcal{L})=O(\delta \cdot h(\mathcal{L})),
$$

as $\delta=1 / N$. This finishes the proof of the lemma.

Combining (20) and Lemma 21, we have

$$
\operatorname{Pr}\left[k_{1}=m-j, k_{2}=j\right]=\sum_{\substack{\mathcal{S}_{1} \subseteq \mathcal{L} \\\left|\mathcal{S}_{1}\right|=m-j}}\left(\prod_{i \in \mathcal{S}_{1}} x_{i, 1} \prod_{i \in \mathcal{L} \backslash \mathcal{S}_{1}}\left(\delta^{i}+\lambda_{i}-x_{i, 1}\right) \prod_{i \notin \mathcal{L}}\left(1-\delta^{i}-\lambda_{i}\right)\right) \pm O(\delta \cdot h(\mathcal{L})) .
$$

The next lemma further simplifies this estimate by absorbing all the $\lambda_{i}$ 's into the error term.

Lemma 22. Given the parameters in (18), we have

$$
\operatorname{Pr}\left[k_{1}=m-j, k_{2}=j\right]=\sum_{\substack{\mathcal{S}_{1} \in \mathcal{L} \\\left|\mathcal{S}_{1}\right|=m-j}}\left(\prod_{i \in \mathcal{S}_{1}} x_{i, 1} \prod_{i \in \mathcal{L} \backslash \mathcal{S}_{1}}\left(\delta^{i}-x_{i, 1}\right) \prod_{i \notin \mathcal{L}}\left(1-\delta^{i}\right)\right) \pm O(\delta \cdot h(\mathcal{L})) .
$$

Proof. First the number of $\mathcal{S}_{1}$ 's is at most $2^{n-1}<N$. Further, fixing an $\mathcal{S}_{1}$ and multiplying out

$$
\prod_{i \in \mathcal{S}_{1}} x_{i, 1} \prod_{i \in \mathcal{L} \backslash \mathcal{S}_{1}}\left(\delta^{i}+\lambda_{i}-x_{i, 1}\right) \prod_{i \notin \mathcal{L}}\left(1-\delta^{i}-\lambda_{i}\right)
$$

will yield $3^{j} \cdot 3^{n-1-m} \leq 3^{n-1}<N^{2}$ many terms. The absolute value of each term with at least one $\lambda_{i}$ must be less than or equal to $\lambda$ because all factors are less than or equal to 1 . There are at most $N^{2}$ many such terms, for each $\mathcal{S}_{1}$, and there are at most $N$ different $\mathcal{S}_{1}$ 's. Using $N^{3} \lambda \ll \delta h(\mathcal{L})$ by (18), we can absorb all terms with at least one $\lambda_{i}$ into the error term $O(\delta \cdot h(\mathcal{L}))$.

Using Lemma 22 and the fact that $\prod_{i \notin \mathcal{L}}\left(1-\delta^{i}\right)>1 / 2$ as $\delta=1 / 2^{n}$, we have

$$
\left(\prod_{i \notin \mathcal{L}}\left(1-\delta^{i}\right)\right)^{-1} \operatorname{Pr}\left[k_{1}=m-j, k_{2}=j\right]=\sum_{\substack{\mathcal{S}_{1} \subseteq \mathcal{L} \\\left|\mathcal{S}_{1}\right|=m-j}}\left(\prod_{i \in \mathcal{S}_{1}} x_{i, 1} \prod_{i \in \mathcal{L} \backslash \mathcal{S}_{1}}\left(\delta^{i}-x_{i, 1}\right)\right) \pm O(\delta \cdot h(\mathcal{L})) .
$$

To understand the RHS better, we define a polynomial $P_{d}^{m}$ for each $d \in[0: m]$ to be

$$
P_{d}^{m}=\sum_{\mathcal{T} \subseteq \mathcal{L},|\mathcal{T}|=d}\left(\prod_{i \in \mathcal{T}} x_{i, 1} \prod_{i \in \mathcal{L} \backslash \mathcal{T}} \delta^{i}\right),
$$

and prove the following lemma that establishes a connection between them. 
Lemma 23. Given $P_{d}^{m}$ defined above, we have

$$
\sum_{\substack{\mathcal{S}_{1} \subseteq \mathcal{L} \\
\left|\mathcal{S}_{1}\right|=m-j}}\left(\prod_{i \in \mathcal{S}_{1}} x_{i, 1} \prod_{i \in \mathcal{L} \backslash \mathcal{S}_{1}}\left(\delta^{i}-x_{i, 1}\right)\right)=\sum_{i=0}^{j}(-1)^{i} \cdot\left(\begin{array}{c}
m-j+i \\
m-j
\end{array}\right) \cdot P_{m-j+i}^{m}
$$

Proof. Note that every monomial that appears on the two sides of (22) has the form $\prod_{i \in \mathcal{T}} x_{i, 1}$ for some $\mathcal{T} \subseteq \mathcal{L}$ with $|\mathcal{T}|=d \geq m-j$. Fix such a $\mathcal{T}$. The coefficient of $\prod_{i \in \mathcal{T}} x_{i, 1}$ on RHS of (22) is

$$
(-1)^{d-m+j} \cdot\left(\begin{array}{c}
d \\
m-j
\end{array}\right) \cdot \prod_{i \in \mathcal{L} \backslash \mathcal{T}} \delta^{i}
$$

On the other hand, for an $\mathcal{S}_{1} \subseteq \mathcal{L}$ with $\left|\mathcal{S}_{1}\right|=m-j$, we have

$$
\prod_{i \in \mathcal{S}_{1}} x_{i, 1} \prod_{i \in \mathcal{L} \backslash \mathcal{S}_{1}}\left(\delta^{i}-x_{i, 1}\right)=\sum_{\mathcal{S}^{\prime} \subseteq \mathcal{L} \backslash \mathcal{S}_{1}}\left(\prod_{i \in \mathcal{S}_{1}} x_{i, 1} \prod_{i \in \mathcal{S}^{\prime}}\left(-x_{i, 1}\right) \prod_{i \in \mathcal{L} \backslash\left\{\mathcal{S}_{1} \cup \mathcal{S}^{\prime}\right\}} \delta^{i}\right) .
$$

Hence, $\prod_{i \in \mathcal{T}} x_{i, 1}$ occurs exactly once in this sum if and only if $\mathcal{S}_{1} \subseteq \mathcal{T}$, and will take the form

$$
\prod_{i \in \mathcal{S}_{1}} x_{i, 1} \prod_{i \in \mathcal{T} \backslash \mathcal{S}_{1}}\left(-x_{i, 1}\right) \prod_{i \in \mathcal{L} \backslash \mathcal{T}} \delta^{i}=(-1)^{d-m+j} \prod_{i \in \mathcal{T}} x_{i, 1} \prod_{i \in \mathcal{L} \backslash \mathcal{T}} \delta^{i}
$$

Further, there are $\left(\begin{array}{c}d \\ m-j\end{array}\right)$ many $\mathcal{S}_{1}$ such that $\mathcal{S}_{1} \subseteq \mathcal{T}$ and $\left|\mathcal{S}_{1}\right|=m-j$. The lemma is proven.

Combining Lemma 22 and 23, we immediately get the following corollary:

Corollary 24. For any $j \in[0: m]$, we have

$$
\left(\prod_{i \notin \mathcal{L}}\left(1-\delta^{i}\right)\right)^{-1} \operatorname{Pr}\left[k_{1}=m-j, k_{2}=j\right]=\sum_{i=0}^{j}(-1)^{i} \cdot\left(\begin{array}{c}
m-j+i \\
m-j
\end{array}\right) \cdot P_{m-j+i}^{m} \pm O(\delta \cdot h(\mathcal{L})) .
$$

Taking a step back, we have derived a set of linear equations that hold with high precision over $\operatorname{Pr}\left[k_{1}=m, k_{2}=0\right], \ldots, \operatorname{Pr}\left[k_{1}=0, k_{2}=m\right]$ and $P_{m}^{m}, \ldots, P_{0}^{m}$. This then allows us to attain a close approximation for $P_{1}^{m}$, using a linear form of the $m$ probabilities. Note that

$$
P_{1}^{m}=\sum_{i \in \mathcal{L}} x_{i, 1} \prod_{j \in \mathcal{L} \backslash\{i\}} \delta^{j}=h(\mathcal{L}) \cdot \sum_{i \in \mathcal{L}} N^{i} \cdot x_{i, 1}
$$

is a linear form of the $x_{i, 1}$ 's, $i \in \mathcal{L}$, including $x_{r, 1}$ (recall that $r$ is the largest integer in $\mathcal{L}$ ). So from here, it will be straightforward to get an approximation of $x_{r, 1}$.

The next lemma gives us a linear form to approximate $P_{1}^{m}$.

Lemma 25. The $m$ probabilities and $P_{1}^{m}$ satisfy

$$
\left(\prod_{i \notin \mathcal{L}}\left(1-\delta^{i}\right)\right)^{-1} \sum_{j=1}^{m} j \cdot \operatorname{Pr}\left[k_{1}=j, k_{2}=m-j\right]=P_{1}^{m} \pm O\left(m^{2} \delta \cdot h(\mathcal{L})\right) .
$$


Proof. By Corollary 24 (and replacing $j$ init by $m-j$ ), we see that it suffices to show that

$$
P_{1}^{m}=\sum_{j=1}^{m} j \cdot\left(\sum_{i=0}^{m-j}(-1)^{i} \cdot\left(\begin{array}{c}
j+i \\
j
\end{array}\right) \cdot P_{j+i}^{m}\right) .
$$

Consider $P_{d}^{m}$ for some $d \in[m] . P_{d}^{m}$ appears in the $j$ th term on the RHS of (24) if and only if $d \geq j$, and when this is the case, the coefficient of $P_{d}^{m}$ is

$$
j \cdot(-1)^{d-j} \cdot\left(\begin{array}{l}
d \\
j
\end{array}\right)
$$

So the RHS of (24) is

$$
\sum_{d=1}^{m} P_{d}^{m} \cdot\left(\sum_{j=1}^{d}(-1)^{d-j} \cdot j \cdot\left(\begin{array}{l}
d \\
j
\end{array}\right)\right)
$$

For $d=1$, the coefficient of $P_{1}^{m}$ is clearly 1 . For $d>1$, using $j\left(\begin{array}{l}d \\ j\end{array}\right)=d\left(\begin{array}{l}d-1 \\ j-1\end{array}\right)$ we have

$$
\sum_{j=1}^{d}(-1)^{d-j} \cdot j \cdot\left(\begin{array}{l}
d \\
j
\end{array}\right)=d \cdot \sum_{j=1}^{d}(-1)^{d-j} \cdot\left(\begin{array}{c}
d-1 \\
j-1
\end{array}\right)=d \cdot \sum_{j=0}^{d-1}(-1)^{d-j-1}\left(\begin{array}{c}
d-1 \\
j
\end{array}\right)=0 .
$$

This finishes the proof of the lemma.

Lemma 25 gives us a linear form to approximate $P_{1}^{m}$. Denote this linear form by $Y_{m}$. Then for the special case when $\mathcal{L}=\{r\}$ (so $r$ is the only integer in $\mathcal{L}$ ), we are done since $P_{1}^{m}$ is exactly $x_{r, 1}$, and we have attained a linear form that approximates $x_{r, 1}$ with error $O\left(m^{2} \delta \cdot h(\mathcal{L})\right)$.

Otherwise suppose $|\mathcal{L}|>1$. We use $r^{\prime}$ to denote the largest integer in $\mathcal{L}$ other than $r$ and write $\mathcal{L}^{\prime}=\left\{i \in \mathcal{S}: i \leq r^{\prime}\right\}\left(\left|\mathcal{L}^{\prime}\right|=m-1\right)$. Repeating the same line of proof so far over $\mathcal{L}^{\prime}$ and $m-1$, we obtain a linear form of $\operatorname{Pr}\left[k_{1}=m-1-j, k_{2}=j\right], j \in[0: m-1]$, denoted by $Y_{m-1}$, to approximate

$$
P_{1}^{m-1}=\sum_{i \in \mathcal{L}^{\prime}} x_{i, 1} \prod_{j \in \mathcal{L}^{\prime} \backslash\{i\}} \delta^{j}=h\left(\mathcal{L}^{\prime}\right) \cdot \sum_{i \in \mathcal{L}^{\prime}} N^{i} \cdot x_{i, 1}
$$

with error $O\left(m^{2} \delta \cdot h\left(\mathcal{L}^{\prime}\right)\right)$. By the definition of $P_{1}^{m}$ and $P_{1}^{m-1}$ in $(23)$ and $(25)$, we have

$$
x_{r, 1}=\delta^{r}\left(\frac{P_{1}^{m}}{h(\mathcal{L})}-\frac{P_{1}^{m-1}}{h\left(\mathcal{L}^{\prime}\right)}\right) .
$$

As a result, we have obtained a linear form

$$
\delta^{r}\left(\frac{Y_{m}}{h(\mathcal{L})}-\frac{Y_{m-1}}{h\left(\mathcal{L}^{\prime}\right)}\right)=x_{r, 1} \pm O\left(m^{2} \delta^{r+1}\right)
$$

over $\operatorname{Pr}\left[k_{1}=m-j, k_{2}=j\right], j \in[0: m]$ and $\operatorname{Pr}\left[k_{, 1}=m-1-j, k_{2}=j\right], j \in[0: m-1]$.

Finally, it follows easily from our derivation of $Y_{m}$ and $Y_{m-1}$ that coefficients of this linear form can be computed in polynomial time in $n$, and every coefficient has absolute value at most $N^{m^{2}}$. 


\section{Membership in PPAD}

In this section we show that $(\alpha, c)$-AnONYMOUS is in PPAD for any constants $\alpha \in \mathbb{N}$ and $c>0$, i.e. the problem of finding an $\epsilon$-approximate equilibrium in an anonymous game $\mathcal{G}=\left(n, \alpha\right.$, payoff $\left.\left._{p}\right\}\right)$ with payoffs from $[0,1]$ is in $\operatorname{PPAD}$, where $\epsilon=1 / 2^{n^{c}}$. Below we use $\operatorname{SIzE}(\mathcal{G})$ to denote the input size of an anonymous game $\mathcal{G}$, i.e., length of the binary representation of $\mathcal{G}$. We write $\operatorname{sizE}(a)$ to denote the length of the binary representation of a rational number $a$, and let $\operatorname{SIZE}(\mathbf{a})=\sum_{i} \operatorname{SIZE}\left(a_{i}\right)$ for a rational vector a (e.g., a rational mixed strategy profile).

Fix constants $\alpha \in \mathbb{N}$ and $c>0$. We show the membership of $(\alpha, c)$-AnONYMOUs by reducing it to a "weak-approximation" fixed point problem [EY10] (see [EY10] for the difference between weak and strong approximations). Given $\mathcal{G}=\left(n, \alpha,\left\{\right.\right.$ payoff $\left.\left._{p}\right\}\right)$, we define a map $F: \Delta \rightarrow \Delta$ (this is the map commonly used to prove the existence of Nash equilibria, e.g., see [Nas51]), where

$$
\Delta=\left\{\left(\mathbf{x}_{i}: i \in[n]\right): \mathbf{x}_{i} \in \mathbb{R}_{+}^{\alpha} \text { is a mixed strategy of player } i \in[n]\right\}
$$

is the set of all mixed strategy profiles. For each $i \in[n]$ and $j \in[\alpha]$, the $(i, j)$ th component of $F$

$$
F_{i, j}(\mathcal{X})=\frac{x_{i, j}+\max \left(0, u_{i}(j, \mathcal{X})-u_{i}(\mathcal{X})\right)}{1+\sum_{k \in[\alpha]} \max \left(0, u_{i}(k, \mathcal{X})-u_{i}(\mathcal{X})\right)},
$$

where $\mathcal{X}=\left(\mathbf{x}_{i}: i \in[n]\right) \in \Delta$ and $\mathbf{x}_{i}=\left(x_{i, 1}, \ldots, x_{i, \alpha}\right)$ for each $i \in[n]$.

Observe that $F$ is continuous and maps $\Delta$ to itself. We also have

Property 26. The map $F$ defined above is polynomial-time computable: Given a rational $\mathcal{X} \in \Delta$, $F(\mathcal{X})$ is rational and can be computed in polynomial time in $\operatorname{SIZE}(\mathcal{G})$ and $\operatorname{sizE}(\mathcal{X})$.

Proof. This follows from the fact that there is a polynomial-time dynamic programming algorithm (see $[\mathrm{DP} 14])$ that computes $u_{i}(j, \mathcal{X})$, given $\mathcal{G}$ and $\mathcal{X}$.

We say $\mathcal{X} \in \Delta$ is an $\epsilon$-approximate fixed point of $F$ if $\|F(\mathcal{X})-\mathcal{X}\|_{\infty} \leq \epsilon$. We prove Lemma 27 in Section 6.1, showing that approximate fixed points of $F$ are approximate Nash equilibria of $\mathcal{G}$.

Lemma 27. Given $\mathcal{X} \in \Delta$ and $0 \leq \epsilon \leq 1$, if $\|F(\mathcal{X})-\mathcal{X}\|_{\infty} \leq \epsilon$, then we have $u_{i}(j, \mathcal{X}) \leq u_{i}(\mathcal{X})+\epsilon^{\prime}$ for all players $i \in[n]$ and pure strategies $j \in[\alpha]$, where $\epsilon^{\prime}=\alpha^{2} \epsilon^{1 / 3}$.

So to find an $\epsilon$-approximate Nash equilibrium $\mathcal{X}$ of $\mathcal{G}$, it suffices to find an $\left(\epsilon^{3} / \alpha^{6}\right)$-approximate fixed point of $F$. Moreover, we show in Section 6.2 that $F$ is polynomially Lipschitz continuous:

Lemma 28. For all $\mathcal{X}, \mathcal{Y} \in \Delta$, we have

$$
\|F(\mathcal{X})-F(\mathcal{Y})\|_{\infty} \leq 10 n \alpha^{n+2} \cdot\|\mathcal{X}-\mathcal{Y}\|_{\infty}
$$

Combining Property 26 and Lemma 28, it follows from Proposition 2.2 (Part 2) of [EY10] that given $\mathcal{G}$ and $\epsilon$ (in binary), the problem of finding an $\epsilon$-approximate fixed point $\mathcal{X}$ of $F$ is in PPAD. The PPAD membership of $(\alpha, c)$-AnONYMOUs then follows from Lemma 27. 


\subsection{Proof of Lemma 27}

For convenience, we write $\max _{i, k}(\mathcal{X})=\max \left(0, u_{i}(k, \mathcal{X})-u_{i}(\mathcal{X})\right)$ for $i \in[n]$ and $k \in[\alpha]$.

In the pursuit of a contradiction, assume that there exist a player $i \in[n]$ and an action $\ell \in[\alpha]$ such that $u_{i}(\ell, \mathcal{X})>u_{i}(\mathcal{X})+\epsilon^{\prime}$. This, along with the fact that $\max _{i, k}(\mathcal{X}) \in[0,1]$, implies that,

$$
\epsilon^{\prime}<\sum_{k \in[\alpha]} \max _{i, k}(\mathcal{X}) \leq \alpha-1 \text {. }
$$

We will show that cases $x_{i, \ell} \leq \alpha \epsilon^{1 / 3}$ and $x_{i, \ell}>\alpha \epsilon^{1 / 3}$ both result in the existence of a strategy $j \in[\alpha]$ such that $\left|F_{i, j}(\mathcal{X})-x_{i, j}\right|>\epsilon$, contradicting our initial assumption.

Case 1: $x_{i, \ell} \leq \alpha \epsilon^{1 / 3}$. Apply (27), (28) and $\epsilon^{\prime}=\alpha^{2} \epsilon^{1 / 3}$ to get

$$
F_{i, \ell}(\mathcal{X})>\frac{x_{i, \ell}+\epsilon^{\prime}}{\alpha} \Rightarrow F_{i, \ell}(\mathcal{X})-x_{i, \ell}>\frac{\epsilon^{\prime}-(\alpha-1) x_{i, \ell}}{\alpha} \geq \frac{\epsilon^{\prime}-(\alpha-1) \alpha \epsilon^{1 / 3}}{\alpha}=\epsilon^{1 / 3} \geq \epsilon .
$$

Case 2: $x_{i, \ell}>\alpha \epsilon^{1 / 3}$. Let $J=\left\{j \in[\alpha]: u_{i}(j, \mathcal{X}) \leq u_{i}(\mathcal{X})\right\}$. We must have

$$
\sum_{j \in J} x_{i, j}\left(u_{i}(\mathcal{X})-u_{i}(j, \mathcal{X})\right) \geq x_{i, \ell}\left(u_{i}(\ell, \mathcal{X})-u_{i}(\mathcal{X})\right)
$$

where $u_{i}(\mathcal{X})-u_{i}(j, \mathcal{X}) \leq 1-\epsilon^{\prime}, u_{i}(\ell, \mathcal{X})-u_{i}(\mathcal{X}) \geq \epsilon^{\prime}$, and $x_{i, \ell}>\alpha \epsilon^{1 / 3}$. Therefore,

$$
\sum_{j \in J} x_{i, j} \geq \frac{\alpha \epsilon^{\prime} \epsilon^{1 / 3}}{1-\epsilon^{\prime}}
$$

which implies that there exists some strategy $j \in J$ such that $x_{i, j} \geq \epsilon^{\prime} \epsilon^{1 / 3} /\left(1-\epsilon^{\prime}\right)$. Apply $(27)$ and $(28)$ to get $F_{i, j}(\mathcal{X})<x_{i, j} /\left(1+\epsilon^{\prime}\right)$, which implies that

$$
\left|F_{i, j}(\mathcal{X})-x_{i, j}\right|>\frac{\epsilon^{\prime} x_{i, j}}{1+\epsilon^{\prime}} \geq \frac{\left(\epsilon^{\prime}\right)^{2} \epsilon^{1 / 3}}{\left(1-\epsilon^{\prime}\right)\left(1+\epsilon^{\prime}\right)} \geq \alpha^{4} \epsilon \geq \epsilon
$$

This finishes the proof of Lemma 27.

\subsection{Proof of Lemma 28}

As $\mathcal{X}-\mathcal{Y}$ is of length $n \alpha$, we have $\|\mathcal{X}-\mathcal{Y}\|_{1} \leq n \alpha \cdot\|\mathcal{X}-\mathcal{Y}\|_{\infty}$. Thus, it suffices to show that

$$
\|F(\mathcal{X})-F(\mathcal{Y})\|_{\infty} \leq 16 \alpha^{n+1}\|\mathcal{X}-\mathcal{Y}\|_{1} .
$$

Fix $i \in[n]$ and $j \in[\alpha]$. We have

$$
\left|F_{i, j}(\mathcal{X})-F_{i, j}(\mathcal{Y})\right|=\left|\frac{x_{i, j}+\max _{i, j}(\mathcal{X})}{1+\sum_{k \in[\alpha]} \max _{i, k}(\mathcal{X})}-\frac{y_{i, j}+\max _{i, j}(\mathcal{Y})}{1+\sum_{k \in[\alpha]} \max _{i, k}(\mathcal{Y})}\right| .
$$

Multiplying the terms in the RHS to get a common denominator, which is clearly $\geq 1$, we get

$$
\begin{aligned}
\left|F_{i, j}(\mathcal{X})-F_{i, j}(\mathcal{Y})\right| \leq & \left|x_{i, j}-y_{i, j}\right|+\left|x_{i, j} \sum_{k \in[\alpha]} \max _{i, k}(\mathcal{Y})-y_{i, j} \sum_{k \in[\alpha]} \max _{i, k}(\mathcal{X})\right| \\
& +\left|\max _{i, j}(\mathcal{X})-\max _{i, j}(\mathcal{Y})\right|+\left|\max _{i, j}(\mathcal{X}) \sum_{k \in[\alpha]} \max _{i, k}(\mathcal{Y})-\max _{i, j}(\mathcal{Y}) \sum_{k \in[\alpha]} \max _{i, k}(\mathcal{X})\right| .
\end{aligned}
$$


To bound $\left|F_{i, j}(\mathcal{X})-F_{i, j}(\mathcal{Y})\right|$, we shall use the following simple trick several times in the rest of the proof. If $a_{1}, a_{2}, b_{1}, b_{2} \in[0,1]$, then we have

$$
\left|a_{1} a_{2}-b_{1} b_{2}\right|=\left|\left(a_{1}-b_{1}\right) a_{2}+b_{1}\left(a_{2}-b_{2}\right)\right| \leq\left|a_{1}-b_{1}\right|+\left|a_{2}-b_{2}\right|,
$$

which easily extends to

$$
\left|a_{1} \cdots a_{n}-b_{1} \cdots b_{n}\right| \leq\left|a_{1}-b_{1}\right|+\cdots+\left|a_{n}-b_{n}\right|,
$$

when all the $a_{i}$ 's and $b_{i}$ 's are in $[0,1]$.

Now we come back to $(29)$. By the definition of $\max _{i, j}(\mathcal{X})$, we have

$$
\begin{aligned}
\left|\max _{i, j}(\mathcal{X})-\max _{i, j}(\mathcal{Y})\right| & \leq\left|\left(u_{i}(j, \mathcal{X})-u_{i}(\mathcal{X})\right)-\left(u_{i}(j, \mathcal{Y})-u_{i}(\mathcal{Y})\right)\right| \\
& \leq\left|u_{i}(j, \mathcal{X})-u_{i}(j, \mathcal{Y})\right|+\left|u_{i}(\mathcal{X})-u_{i}(\mathcal{Y})\right|
\end{aligned}
$$

As $\mathcal{X}, \mathcal{Y} \in \Delta$ we have $x_{i, j}, y_{i, j} \in[0,1]$. Since all payoffs of $\mathcal{G}$ are in $[0,1]$, we have $u_{i}(j, \mathcal{X}), u_{i}(j, \mathcal{Y})$, $u_{i}(\mathcal{X}), u_{i}(\mathcal{Y}) \in[0,1]$ for all $i, j$, which in turn implies that $\max _{i, j}(\mathcal{X}), \max _{i, j}(\mathcal{Y}) \in[0,1]$.

Using these properties above, along with the trick, we can conclude

$$
\begin{aligned}
\mid x_{i, j} \sum_{k \in[\alpha]} \max _{i, k}(\mathcal{Y}) & -y_{i, j} \sum_{k \in[\alpha]} \max _{i, k}(\mathcal{X})\left|\leq \sum_{k \in[\alpha]}\right| x_{i, j} \cdot \max _{i, k}(\mathcal{Y})-y_{i, j} \cdot \max _{i, k}(\mathcal{X}) \mid \\
& \leq \sum_{k \in[\alpha]}\left(\left|x_{i, j}-y_{i, j}\right|+\left|u_{i}(k, \mathcal{X})-u_{i}(k, \mathcal{Y})\right|+\left|u_{i}(\mathcal{X})-u_{i}(\mathcal{Y})\right|\right)
\end{aligned}
$$

Similarly, we also have

$$
\begin{aligned}
\left|\max _{i, j}(\mathcal{X}) \sum_{k \in[\alpha]} \max _{i, k}(\mathcal{Y})-\max _{i, j}(\mathcal{Y}) \sum_{k \in[\alpha]} \max _{i, k}(\mathcal{X})\right| \\
\leq \sum_{k \in[\alpha]}\left(\left|u_{i}(j, \mathcal{X})-u_{i}(j, \mathcal{Y})\right|+2\left|u_{i}(\mathcal{X})-u_{i}(\mathcal{Y})\right|+\left|u_{i}(k, \mathcal{X})-u_{i}(k, \mathcal{Y})\right|\right) .
\end{aligned}
$$

Plugging all these back into (29), we have

$$
\begin{aligned}
\left|F_{i, j}(\mathcal{X})-F_{i, j}(\mathcal{Y})\right| \leq(1+\alpha) \cdot\left|x_{i, j}-y_{i, j}\right|+(1+3 \alpha) \cdot\left|u_{i}(\mathcal{X})-u_{i}(\mathcal{Y})\right| \\
+(1+\alpha) \cdot\left|u_{i}(j, \mathcal{X})-u_{i}(j, \mathcal{Y})\right|+2 \cdot \sum_{k \in[\alpha]}\left|u_{i}(k, \mathcal{X})-u_{i}(k, \mathcal{Y})\right|
\end{aligned}
$$

Finally, we bound $\left|u_{i}(k, \mathcal{X})-u_{i}(k, \mathcal{Y})\right|$ in terms of $\|\mathcal{X}-\mathcal{Y}\|_{1}$. Let $S$ be the set of pure strategy profiles. Then, by applying the trick and the fact that all payoffs are in $[0,1]$, it follows that

$$
\begin{aligned}
\left|u_{i}(k, \mathcal{X})-u_{i}(k, \mathcal{Y})\right| & \leq \sum_{\mathbf{s} \in S_{-i}}\left|\prod_{q \neq i} x_{q, s_{q}}-\prod_{q \neq i} y_{q, s_{q}}\right| \leq \sum_{\mathbf{s} \in S_{-i}} \sum_{q \neq i}\left|x_{q, s_{q}}-y_{q, s_{q}}\right| \leq \alpha^{n-1}\|\mathcal{X}-\mathcal{Y}\|_{1} \\
\left|u_{i}(\mathcal{X})-u_{i}(\mathcal{Y})\right| & \leq \sum_{\mathbf{s} \in S}\left|\prod_{q \in[n]} x_{q, s_{q}}-\prod_{q \in[n]} y_{q, s_{q}}\right| \leq \sum_{\mathbf{s} \in S} \sum_{q \in[n]}\left|x_{q, s_{q}}-y_{q, s_{q}}\right| \leq \alpha^{n}\|\mathcal{X}-\mathcal{Y}\|_{1} .
\end{aligned}
$$


Applying these inequalities, along with $\left|x_{i, j}-y_{i, j}\right| \leq\|\mathcal{X}-\mathcal{Y}\|_{1}$, we get

$$
\left|F_{i, j}(\mathcal{X})-F_{i, j}(\mathcal{Y})\right| \leq 10 \alpha^{n+1} \cdot\|\mathcal{X}-\mathcal{Y}\|_{1}
$$

This finishes the proof Lemma 28.

\section{Open Problems}

Can the number of strategies be further reduced from seven in our PPAD-hardness result? Specifically, could we construct an anonymous game similar to the radix game $\mathcal{G}_{n, N}$, particularly its set of approximate Nash equilibria after perturbation, but without the four special (auxiliary) pure strategies $\left\{q_{1}, q_{2}, r_{1}, r_{2}\right\}$ ? While we believe this to be possible, constructing such a game can be highly non-trivial and would require specifying different payoffs for many of the possible outcomes seen by each player. Accordingly, proving a result similar to Lemma 11 after duplicating the first strategy would be even more difficult.

However, even the construction of such a game would only reduce the number of strategies used in the hardness proof down to three (due to the strategy duplication in the generalized radix game later), leading to the next open question: Is there an FPTAS for two-strategy anonymous games? As was posited by Daskalakis and Papadimitriou, it remains unclear whether a rational two-strategy anonymous game always has a rational Nash equilibrium. Additionally, in their sequence of paper's proving a PTAS for a bounded number of strategies, Daskalakis and Papadimitriou found that the form of the $\operatorname{Pr}_{\mathcal{X}}[p, \mathbf{k}]$ is significantly simpler for two-strategy anonymous games. Correspondingly, we found that constructing useful gadgets for reductions with just two strategies to be very difficult, suggesting that an FPTAS for two-strategy anonymous games is certainly a possibility.

Moreover, could there be an FPTAS for anonymous games with any bounded number of pure strategies? There is no clear way to strengthen our current construction to obtain a PPAD-hardness result for $1 / \operatorname{poly}(n)$-approximate Nash equilibrium. In order for the estimation lemma to hold, we need $x_{i, 1}+x_{i, 2} \approx \delta^{i}$ for all $i$. So even if we set $N=2$, ensuring that $x_{i, 1}+x_{i, 2}=\delta^{i} \pm O(1 / \operatorname{poly}(n))$ would still not be sufficient for the estimation lemma to hold. Accordingly, in order to modify our construction to get such a hardness result, we would need to construct an anonymous game, which

contains $n$ players with the same properties as the main players in the generalized radix game, but with the additional property that $O(1 / \operatorname{poly}(n))$ shifts in the payoffs would only cause $O\left(1 / 2^{\text {poly }(n)}\right)$ shifts in $x_{i, 1}+x_{i, 2}$, which seems incredibly unlikely.

\section{References}

[ABOV07] L. Addario-Berry, N. Olver, and A. Vetta. A polynomial time algorithm for finding Nash equilibria in planar win-lose games. Journal of Graph Algorithms and Applications, 11(1):309-319, 2007.

[AKV05] T. Abbott, D. Kane, and P. Valiant. On the complexity of two-player win-lose games. In Proceedings of the 46th Annual IEEE Symposium on Foundations of Computer Science, pages 113-122, 2005.

[ARV08] H. Ackermann, H. Röglin, and B. Vöcking. On the impact of combinatorial structure on congestion games. Journal of the ACM, 55:1-22, 2008. 
[AS11] Y. Azrieli and E. Shmaya. Lipschitz games. Mimeo, Ohio State University, 2011.

[Bab13] Y. Babichenko. Best-reply dynamics in large binary-choice anonymous games. Games and Economic Behavior, 81:130-144, 2013.

[BBM07] H. Bosse, J. Byrka, and E. Markakis. New algorithms for approximate Nash equilibria in bimatrix games. In Proceedings of the 3rd International Workshop on Internet and Network Economics, pages 17-29, 2007.

[BFH09] F. Brandt, F. Fischer, and M. Holzer. Symmetries and the complexity of pure Nash equilibrium. Journal of Computer and System Sciences, 75(3):163-177, 2009.

[Blo99] M. Blonski. Anonymous games with binary actions. Games and Economic Behavior, pages 171-180, 1999.

[Blo05] M. Blonski. The women of Cairo: Equilibria in large anonymous games. Journal of Mathematical Economics, pages 254-263, 2005.

[BVV05] I. Bárány, S. Vempala, and A. Vetta. Nash equilibria in random games. In Proceedings of the 46th Annual IEEE Symposium on Foundations of Computer Science, pages 123-131, 2005.

[CD11] Y. Cai and C. Daskalakis. On minmax theorems for multiplayer games. In Proceedings of the 22nd ACM-SIAM Symposium on Discrete Algorithms, pages 217-234, 2011.

[CDT06] X. Chen, X. Deng, and S.-H. Teng. Sparse games are hard. In Proceedings of the 2nd Workshop on Internet and Network Economics, pages 262-273, 2006.

[CDT09] X. Chen, X. Deng, and S.-H. Teng. Settling the complexity of computing two-player Nash equilibria. Journal of the ACM, 56(3):1-57, 2009.

[CFGS11] I. Caragiannis, A. Fanelli, N. Gravin, and A. Skopalik. Efficient computation of approximate pure Nash equilibria in congestion games. In Proceedings of the 52nd Annual IEEE Symposium on Foundations of Computer Science, pages 532-541, 2011.

[CFGS12] I. Caragiannis, A. Fanelli, N. Gravin, and A. Skopalik. Approximate pure Nash equilibria in weighted congestion games: existence, efficient computation, and structure. In Proceedings of the 13th ACM Conference on Electronic Commerce, 2012.

[CPY13] X. Chen, D. Paparas, and M. Yannakakis. The complexity of non-monotone markets. In Proceedings of the 45th Annual ACM Symposium on Theory of Computing, pages 181-190, 2013.

[CS11] S. Chien and A. Sinclair. Convergence to approximate Nash equilibria in congestion games. Games and Economic Behavior, 71(2):315-327, 2011.

[CTV07] X. Chen, S.-H. Teng, and P. Valiant. The approximation complexity of win-lose games. In Proceedings of the 18th Annual ACM-SIAM Symposium on Discrete Algorithms, pages 159-168, 2007. 
[Das08] C. Daskalakis. An efficient PTAS for two-strategy anonymous games. In Proceedings of the 4th International Workshop on Internet and Network Economics, pages 186-197, 2008.

[DGP09] C. Daskalakis, P.W. Goldberg, and C.H. Papadimitriou. The complexity of computing a Nash equilibrium. SIAM Journal on Computing, 39(1), 2009.

[DMP06] C. Daskalakis, A. Mehta, and C.H. Papadimitriou. A note on approximate Nash equilibria. In Proceedings of the 2nd Workshop on Internet and Network Economics, pages 297-306, 2006.

[DMP07] C. Daskalakis, A. Mehta, and C.H. Papadimitriou. Progress in approximate Nash equilibria. In Proceedings of the 8th ACM Conference on Electronic Commerce, pages 355-358, 2007.

[DP07] C. Daskalakis and C.H. Papadimitriou. Computing equilibria in anonymous games. In Proceedings of the 48th Annual IEEE Symposium on Foundations of Computer Science, pages 83-93, 2007.

[DP08] C. Daskalakis and C.H. Papadimitriou. Discretized multinomial distributions and Nash equilibria in anonymous games. In Proceedings of the 49 th Annual IEEE Symposium on Foundations of Computer Science, pages 25-34, 2008.

[DP09] C. Daskalakis and C.H. Papadimitriou. On oblivious PTAS's for Nash equilibrium. In Proceedings of the 41st Annual ACM Symposium on Theory of Computing, pages 75-84, 2009.

[DP14] C. Daskalakis and C.H. Papadimitriou. Approximate Nash equilibria in anonymous games. Journal of Economic Theory, 2014.

[ES05] J.C. Ely and W.H. Sandholm. Evolution in Bayesian games I. Theory of Games and Economic Behavior, 53:83-109, 2005.

[EY10] K. Etessami and M. Yannakakis. On the complexity of Nash equilibria and other fixed points. SIAM Journal on Computing, 39(6):2531-2597, 2010.

[FNS07] T. Feder, H. Nazerzadeh, and A. Saberi. Approximating Nash equilibria using small-support strategies. In Proceedings of the 8th ACM Conference on Electronic Commerce, pages 352-354, 2007.

[FPT04] A. Fabrikant, C.H. Papadimitriou, and K. Talwar. The complexity of pure Nash equilibria. In Proceedings of the 36th Annual ACM Symposium on Theory of Computing, pages 604-612, 2004.

[HR04] C.A. Holt and A.E. Roth. The Nash equilibrium: A perspective. Proceedings of the National Academy of Sciences, 101(12):3999-4002, 2004.

[Kal05] E. Kalai. Partially-specified large games. Proceedings of the 1st International Workshop on Internet and Network Economics, pages 3-13, 2005. 
[KPS06] S. Kontogiannis, P. Panagopoulou, and P. Spirakis. Polynomial algorithms for approximating Nash equilibria of bimatrix games. In Proceedings of the 2nd Workshop on Internet and Network Economics, pages 286-296, 2006.

[KS07] S.C. Kontogiannis and P.G. Spirakis. Efficient algorithms for constant well supported approximate equilibria in bimatrix games. In Proceedings of the 34 th International Colloquium on the Automata, Languages and Programming, pages 595-606, 2007.

[KT07] R. Kannan and T. Theobald. Games of fixed rank: A hierarchy of bimatrix games. In Proceedings of the 18th Annual ACM-SIAM Symposium on Discrete Algorithms, pages 1124-1132, 2007.

[LMM04] R.J. Lipton, E. Markakis, and A. Mehta. Playing large games using simple strategies. In Proceedings of the 4th ACM Conference on Electronic Commerce, pages 36-41, 2004.

[Meh14] R. Mehta. Constant rank bimatrix games are PPAD-hard. In Proceedings of the 46th Annual ACM Symposium on Theory of Computing, pages 545-554, 2014.

[Mil96] I. Milchtaich. Congestion games with player-specific payoff functions. Games and Economic Behavior, pages 111-124, 1996.

[Nas50] J.F. Nash. Equilibrium points in n-person games. Proceedings of the National Academy of Sciences, 36(1):48-49, 1950.

[Nas51] J.F. Nash. Non-cooperative games. Annals of Mathematics, 54(2):286-295, 1951.

[PR08] C.H. Papadimitriou and T. Roughgarden. Computing correlated equilibria in multi-player games. Journal of the ACM, 55(3):1-29, 2008.

[Ras83] S. Rashid. Equilibrium points of nonatomic games: Asymptotic results. Economics Letters, 12:7-10, 1983.

[Ros73] R.W. Rosenthal. A class of games possessing pure-strategy Nash equilibria. International Journal of Game Theory, 2(1):65-67, 1973.

[Sch73] D. Schmeidler. Equilibrium points of nonatomic games. Journal of Statistical Physics, $7(4): 295-300,1973$.

[SV08] A. Skopalik and B. Vöcking. Inapproximability of pure Nash equilibria. In Proceedings of the 40th Annual ACM Symposium on Theory of Computing, pages 355-364, 2008.

[TS07] H. Tsaknakis and P.G. Spirakis. An optimization approach for approximate Nash equilibria. In Proceedings of the 3rd International Workshop on Internet and Network Economics, pages 42-56, 2007.

[TS10] H. Tsaknakis and P.G. Spirakis. Practical and efficient approximations of Nash equilibria for win-lose games based on graph spectra. In Proceedings of the 6th International Conference on Internet and Network Economics, pages 378-390, 2010. 